\title{
THE CFHTLS-DEEP CATALOG OF INTERACTING GALAXIES. I. MERGER RATE EVOLUTION TO $z=1.2$
}

\author{
C. R. Bridge ${ }^{1,2}$, R. G. CARLBERG ${ }^{2}$, AND M. Sullivan ${ }^{2,3}$ \\ ${ }^{1}$ California Institute of Technology, Pasadena, CA 91125, USA; bridge@ astro.caltech.edu \\ ${ }^{2}$ University of Toronto, 50 St. George Street, Toronto, ON M5S 3H4, Canada \\ ${ }^{3}$ Department of Astrophysics, University of Oxford, Keble Road, Oxford OX1 3RH, UK \\ Received 2009 March 31; accepted 2009 December 9; published 2010 January 11
}

\begin{abstract}
We present the rest-frame optical galaxy merger fraction between $0.2<z<1.2$, as a function of stellar mass and optical luminosity, as observed by the Canada-France-Hawaii Telescope Legacy Deep Survey (CFHTLS-Deep). We developed a new classification scheme to identify major galaxy-galaxy mergers based on the presence of tidal tails and bridges. These morphological features are signposts of recent and ongoing merger activity. Through the visual classification of all galaxies, down to $i_{\text {vega }} \leqslant 22.2(\approx 27,000$ galaxies) over 2 square degrees, we have compiled the CFHTLS-Deep Catalog of Interacting Galaxies, with $\approx 1600$ merging galaxies. We find the merger fraction to be $4.3 \% \pm 0.3 \%$ at $z \sim 0.3$ and $19.0 \% \pm 2.5 \%$ at $z \sim 1$, implying evolution of the merger fraction going as $(1+z)^{m}$, with $m=2.25 \pm 0.24$. This result is inconsistent with a mild or non-evolving $(m<1.5)$ scenario at a $\gtrsim 4 \sigma$ level of confidence. A mild trend, where by massive galaxies with $M_{*}>10^{10.7} M_{\odot}$ are undergoing fewer mergers than less massive systems $\left(M_{*} \sim 10^{10} M_{\odot}\right)$, consistent with the expectations of galaxy assembly downsizing is observed. Our results also show that interacting galaxies have on average SFRs double that found in non-interacting field galaxies. We conclude that (1) the optical galaxy merger fraction does evolve with redshift, (2) the merger fraction depends mildly on stellar mass, with lower mass galaxies having higher merger fractions at $z<1$, and (3) star formation is triggered at all phases of a merger, with larger enhancements at later stages, consistent with $N$-body simulations.
\end{abstract}

Key words: galaxies: evolution - galaxies: formation - galaxies: interactions - galaxies: starburst

Online-only material: color figures

\section{INTRODUCTION}

In the past three decades, the concept of galaxies as "island universes" slowly evolving in isolation has changed dramatically. Gravitational interaction between galaxies is now considered a relevant factor in a galaxy's evolution, capable of altering its morphology, luminosity, color, size, star formation rate (SFR), and mass distribution, all over a relatively short timescale. According to the $\Lambda$-dominated cold dark matter $(\Lambda \mathrm{CDM})$ model, the merger rate of dark matter halos and similarly the merger rate of galaxies are the most fundamental processes in structure formation. Numerous $N$-body simulations and semi-analytical models have studied the merger rate of dark matter halos, predicting evolution with redshift as $(1+z)^{m}$, with $1.0<m<3.5$ (Gottlöber et al. 2001; Berrier et al. 2006; Fakhouri \& Ma 2008). A direct comparison between dark matter halo merger rates and galaxy merger rates is difficult due to the uncertainty in the galaxy halo occupation number. Although measuring the frequency that galaxies merge as a function of cosmic epoch can place powerful constraints on the theory of galaxy evolution and structure formation.

Traditional observational approaches aimed at investigating the major galaxy merger rate, measure the frequency of galaxy mergers or galaxies in close pairs, spanning a range of redshifts. The merger or close pair fraction should evolve in a similar manner as the merger rate assuming the timescale over which the merger selection criteria are sensitive to is independent of redshift. Toomre (1977) was the first to suggest that the merger rate may be larger at higher redshifts, by using estimates of past merger remnants. Numerous studies have estimated the evolution of the merger rate, yielding highly discrepant measurements of $m$, ranging from no evolution $(m \sim 0)$ to strong evolution $(m \sim 5$; Zepf \& Koo 1989; Carlberg et al.
1994, 2000; Patton et al. 1997; Le Fèvre et al. 2000; Conselice et al. 2003; Lin et al. 2004; Bridge et al. 2007; Kartaltepe et al. 2007; Lotz et al. 2008b, to name a few). It is often suggested that this discrepancy stems from the variety of techniques used to identify galaxy mergers, incompleteness corrections, as well as differences in sample selection. Although these factors likely play a role, typical sample sizes have been on the order of a few tens of mergers (excluding Kartaltepe et al. 2007) resulting in $>30 \%$ uncertainty in the evolution of the merger rate, and the effect of cosmic variance has been largely unexplored.

Over the past decade it has become clear that the average SFR per unit comoving volume (CSFR) has declined by an order of magnitude since $z \sim 1$ (Lilly et al. 1996; Madau et al. 1998). A fundamental question that remains is the cause of the drop of the CSFR. It has been suggested by some close pair (Burkey et al. 1994; Yee \& Ellingson 1995; Patton et al. 1997, 2002; Bridge et al. 2007; Kartaltepe et al. 2007) and merger studies (Le Fèvre et al. 2000; Conselice et al. 2003; Kampczyk et al. 2007) that an increased merger rate at higher redshift is either partially or completely responsible for the higher SFR density at $z \sim 1$. However, there are merger studies which have found little or no evolution of the merger rate (Carlberg et al. 2000; Bundy et al. 2004; Lin et al. 2004; Lotz et al. 2008b), indicating the decrease in the volume-averaged SFR density since $\sim 1$ is not a result of a declining merger rate but rather declining star formation in disk galaxies (Lotz et al. 2008b).

In order to probe the merger rate a clear definition of what constitutes a merger and a robust identification technique are required. One traditional method is to search for close galaxy pairs. However, even with radial velocity measurements for both galaxies, about half of all physical pairs may be chance superpositions (Patton et al. 2000, 2002). Another method is to calculate internal asymmetries, but these quantitative morphological 
parameters are subject to complication, since star formation itself is sufficiently violent and chaotic and therefore not smoothly distributed, potentially mimicking features of a merger when none has occurred. However, long tidal tails are nearly a foolproof signature of imminent mergers. Moreover, tidal tails are a relatively simple, completely dynamical phenomenon that can be studied in detail with $N$-body simulations (Toomre \& Toomre 1972; Barnes 1992; Hopkins et al. 2008). In spite of these positive features, little work on galaxies with tidal tails has been done at high redshift. The reason is simple: tails have a relatively low surface brightness.

The Deep component of the Canada France Hawaii Legacy Survey (hereafter CFHTLS-Deep) provides some unique advantages for studying morphologies and the merger rate of galaxies. The deep ground-based imaging $\left(i^{\prime}<27.3\right)$ is ideal for the detection of low surface brightness features like tidal tails. The survey area is also spread over four fields allowing for cosmic variance estimates of the merger rate, while the larger area allows for merger fraction estimates no longer dominated by small number statistics as seen in previous studies (excluding Kartaltepe et al. 2007).

In this paper, we apply a new technique for identifying major merger candidates in CFHTLS-Deep. Through the visual classification of $\approx 27,000$ galaxies over 2 square degrees, we have complied the CFHTLS-Deep Catalog of Interacting Galaxies, which contains $\approx 1600$ galaxies between $0.1<z<1.2$ with tidal tails and bridges. This is the largest catalog of its kind in the literature. In Section 2, we describe the optical photometry from CFHTLS-Deep, along with the methodology used for deriving the photometric redshifts, stellar masses and SFRs. Section 3 outlines the technique used to identify interacting galaxies, including completeness tests. We measure the galaxy interaction fraction in Section 4, its dependence on optical luminosity, stellar mass, and address cosmic variance. With additional assumptions about the merger timescale, we estimate the merger rate and interaction history of galaxies from $z \sim 1$ in Section 5, followed by the impact mergers have on the SFRs of galaxies (Section 6). We summarize our conclusions in Section 7.

In the discussion that follows, we assume Vega magnitudes and any calculation requiring cosmology assumes $\Omega_{\mathrm{M}}=$ $0.3, \Omega_{\Lambda}=0.70$, and $H_{0}=70 \mathrm{~km} \mathrm{~s}^{-1} \mathrm{Mpc}^{-1}$.

\section{THE CFHTLS-DEEP OBSERVATIONS}

\subsection{MegaCam Optical Imaging}

The CFHT Legacy Survey (CFHTLS) is a joint community project between Canada and France with more than 450 nights over a 5 year period that commenced in 2003 June. The survey exploits the square degree MegaCam camera (Boulade et al. 2003) on CFHT which has 36, $2048 \times 4612$ pixel CCDs with a pixel scale of 0'.187. The Deep survey is one component of the CFHTLS (deep, wide, very wide), covering four low Galactic extinction fields in $u^{*}, g^{\prime}, r^{\prime}, i^{\prime}, z^{\prime}$. Each survey field, named D1 through D4 is one square degree and distributed in right ascension (R.A.) for efficient observing throughout the year. The survey fields were centered at J2000 R.A. $=02^{\mathrm{h}} 26^{\mathrm{m}} 00^{\mathrm{s}}$, decl. $=-04^{\mathrm{deg}} 30^{\prime} 00^{\prime \prime}$ (D1), R.A. $=10^{\mathrm{h}} 00^{\mathrm{m}} 29^{\mathrm{s}}$, decl. $=+02^{\mathrm{deg}} 12^{\prime} 21^{\prime \prime}$ (D2), R.A. $=$ $14^{\mathrm{h}} 17^{\mathrm{m}} 54^{\mathrm{s}}$, decl. $=+52^{\mathrm{deg}} 30^{\prime} 31^{\prime \prime}\left(\right.$ D3), R.A. $=22^{\mathrm{h}} 15^{\mathrm{m}} 31^{\mathrm{s}}$, decl. $=-17^{\mathrm{deg}} 44^{\prime} 05^{\prime \prime}$ (D4). The analysis that follows utilizes only the D1 and D2 fields, a total of 2 square degrees, due to their extensive ancillary data (see Sullivan et al. 2006a, for details).
Table 1

CFHTLS-Deep: Final Stacks

\begin{tabular}{lccc}
\hline \hline \multicolumn{1}{c}{ Field } & Filter & Int. Time $(\mathrm{hr})$ & Limiting Magnitude \\
\hline D1.......... & $u^{*}$ & 10.6 & 26.7 \\
(0.959 square degree) & $g^{\prime}$ & 9.5 & 27.1 \\
& $r^{\prime}$ & 18.8 & 26.8 \\
& $i^{\prime}$ & 45.1 & 26.4 \\
& $z^{\prime}$ & 20.0 & 25.6 \\
\hline D2......... & $u^{*}$ & 3.4 & 26.4 \\
$(0.900$ square degree) & $g^{\prime}$ & 5.7 & 27.0 \\
& $r^{\prime}$ & 10.7 & 26.5 \\
& $i^{\prime}$ & 22.2 & 26.2 \\
& $z^{\prime}$ & 12.0 & 25.2 \\
\hline
\end{tabular}

Note. Limiting magnitudes are estimated by adding artificial galaxies, faded and rescaled into the images and then trying to recover them.

The optical images used to derive the galaxy parameters and morphological classification were constructed by the Supernova Legacy Survey (SNLS; Sullivan et al. 2006b), a key project in the CFHTLS. Deep optical stacks were generated for each filter $\left(u^{*}, g^{\prime}, r^{\prime}, i^{\prime}, z^{\prime}\right)$ with an imposed wavelength-dependent seeing limit $(\sim 4$ pixels) to ensure a high degree of resolution. The goal was to maximize exposure depth while retaining similar and excellent seeing in the different filters. The typical seeing of the final stacks is $0.7-0$ '. 8 ( $i^{\prime}$ band). The individual "Elixir" processed images are available from the Canadian Astronomy Data Centre (CADC); however, the final stacks and photometric catalogs including redshifts in which this work is based are not currently public. The details of the processing technique are discussed in (Sullivan et al. 2006b).

Source extraction and photometry were performed on each Deep field using SExtractor (Bertin \& Arnouts 1996) in dual image mode. Detections were performed in the $i^{\prime}$ filter $\left(i^{\prime} \sim 26.3\right)$ and photometry measurements calculated in each of the five filters, $u^{*}, g^{\prime}, r^{\prime}, i^{\prime}, z^{\prime}$. The exposures times and limiting magnitudes reached for each filter in both fields are given in Table 1. We applied a bright star and bad pixel mask to the images prior to running the source detection to eliminate noisy or contaminated regions. The total area masked is $\leqslant 10 \%$ for each field. We compared our number counts to those from the Hubble Deep Field (HDF) North and South (Williams et al. 1996; Metcalfe et al. 2001) and COSMOS (Leauthaud et al. 2007). They were found to be consistent for galaxies brighter than $i^{\prime}<24$, on which this work is focused.

\subsection{Galaxy Properties: Redshift, Stellar Mass, SFRs}

In this section, we outline the methods used to convert the optical fluxes of our sources into a photometric redshift, and derive other properties such as stellar mass and SFR.

In order to study the potential evolution of the interaction and SFRs of galaxies, we need to derive a redshift estimate for each galaxy. Although spectroscopic redshifts are the most precise distance measures, they are observationally expensive to obtain for large samples. A strength of CFHTLS-Deep is its high quality five-band optical imaging, which can be used to derive a photometric redshift estimate. The five optical bands can be combined to produce a broadband spectral energy distribution (SED) that can be compared to a set of template SEDs to estimate properties of the galaxy such as its redshift, age, stellar mass, and SFR (Baum 1962; Loh \& Spillar 1986). This technique uses broad spectral features like the $4000 \AA$ discontinuity or the Lyman break for comparison to templates. 
We fit a series of galaxy template SEDs to the broadband fluxes of each galaxy. The best-fit SED is determined through a standard $\chi^{2}$ minimization procedure between the synthetic photometry generated by integrating the template SEDs through the CFHTLS filters, and the observed fluxes (including the flux errors). This was performed with the Z-Peg template fitting code (Bolzonella et al. 2000; Le Borgne \& Rocca-Volmerange 2002). Its methodology is similar to that applied by other photometric redshift codes (e.g., Gwyn \& Hartwick 1996).

We employ a set of synthetic templates computed with the PEGASE-II galaxy evolution code (Fioc \& Rocca-Volmerange 1997; Le Borgne \& Rocca-Volmerange 2002; Le Borgne et al. 2004). Both the SEDs and the photometric redshift code have been extensively tested and used in the literature (e.g., McCarthy et al. 2004; Grazian et al. 2006; Sullivan et al. 2006b). We use eight evolutionary scenarios that evolve self-consistently with age and assume a Rana-Basu initial mass function (Rana \& Basu 1992). These scenarios were designed to match the average colors of local galaxies and to reproduce deep galaxy number counts. Considering several tens of time steps for each scenario, the total number of synthetic SEDs is $\approx 500$. When fitting a given galaxy only templates younger than the age of the universe at the redshift of the galaxy are considered. The accuracy of the photometric redshifts is determined by comparing them to the SNLS spectroscopic sample in our fields (Howell et al. 2005; Bronder et al. 2008). The photometric redshift accuracy down to $i^{\prime} \sim 22.5$ is $\sigma_{\Delta z} /\left(1+z_{s}\right)=0.04$.

Two additional physical parameters of particular interest are the SFRs of interacting galaxies and their stellar masses. These quantities are both derived using the Z-Peg code (Le Borgne \& Rocca-Volmerange 2002). The mean recent SFR for a galaxy is determined from the best-fit SED, averaging the SFR over a period of 0.5 Gyr. The total stellar mass of a galaxy was derived by integrating the total star formation history (SFH) of the best-fit scenario, up to the best-fit age and subtracting off the mass from stars that have died. The results from these techniques were found to be in good agreement with template fits when a spectroscopic redshift was used (Sullivan et al. 2006b). The systematic errors associated with these techniques, their application to CFHTLS data, and consistency checks are outlined in Sullivan et al. (2006b).

\section{CFHTLS-DEEP CATALOG OF INTERACTING GALAXIES}

\subsection{Identifying Interacting Galaxies}

The first step in studying the frequency of galaxies involved in an interaction is to define a clean, robust, and useful definition of an interacting galaxy. Morphologically, interacting galaxies can exhibit long tidal tails, bridges (linking two or more galaxies), ring structures, stellar bars and/or enhanced spiral structure and frequently appear severely distorted. We have focused our identification methods on confidently selecting galaxies which have recently undergone or are presently undergoing a tidal interaction. The presence of a tidal tail or bridge is incontrovertible evidence of a recent interaction.

There are two different avenues one can take to morphologically select interacting galaxies. The first is to utilize quantitative morphological software that measures a galaxy's structural parameters, such as asymmetry, Gini or $M_{20}$ (Abraham et al. 1996a, 1996b; Conselice et al. 2000; Lotz et al. 2004). As a galaxy undergoes a merger, tidal fields distort the galaxies radially, drawing out galactic material into long plumes and tails.
These structural parameters can help describe the level of a galaxy's disturbed appearance and are commonly used to identify mergers.

This is an efficient and automated approach. However, a complication of this method is the need for high-resolution data, especially at high redshifts when Hubble Space Telescope (HST) observations are a requirement. A further complication is that $H S T /$ ACS images do not easily detect low surface brightness features, like tidal tails due in combination to the small plate scale and point spread function (PSF).

The second method of identifying interacting galaxies is based on pure visual inspection. This technique has been utilized by many in the past to morphologically classify galaxies (Hubble 1926; Vorontsov-Velyaminov 1959; Sandage 1961; Arp 1966, to name a few). Qualitative classification is able to identify the low surface brightness features that the automated method has difficulties with, as it utilizes one of the best pattern recognition computers - the brain-eye combination. A drawback of this method is that it can be laborious and subjective and can suffer from reproducibility issues when the person conducting the classification changes. However, when a set of visual criteria are clearly defined, and the features themselves are striking (e.g., long tidal tails and bridges), visual inspection can be a highly accurate method of morphological classification (see Section 3.3).

The ground-based nature of our data set coupled with its depth makes it ideal to detect low surface brightness features down to $<29$ mag $\operatorname{arcsec}^{-2}$. Ultimately, we define an interacting galaxy to be one with a tidal tail or bridge. These merger signatures are visible after a first encounter and can persist even after the galaxies' nuclei have coalesced. Although our primary objective was to identify galaxies undergoing an interaction, other classifications were included and will be presented in a future paper.

The morphology of galaxy interactions relies on a large number of variables, such as the geometry of the encounter, impact velocity, and mass ratios (Barnes 1992). By defining a sample of tidal tailed galaxies, we are selecting galaxy mergers which have recently experienced a merger of mass ratio $M_{1} / M_{2}>1 / 10$. Galaxy encounters with mass ratios $>1 / 10$ have been shown to have significant impact on a galaxy's evolution (Barnes 1992; Mihos \& Hernquist 1996). Although some of the tailed galaxies in our sample will have been a result of lower mass ratio or minor mergers (defined as those with $\left.1 / 10<M_{1} / M_{2}<1 / 4\right)$, the majority are likely to have been the consequence of $M_{1} / M_{2}>1 / 4$ interactions, due to the marked nature of the tidal features before being included in our sample (e.g., Barnes 1992; Mihos \& Hernquist 1996; Dubinski et al. 1999).

\subsection{Classification Methodology and Scheme}

The large area and deep optical imaging of CFHTLS-Deep makes it well suited to identifying interacting galaxies. All galaxies in the D1 and D2 fields (2 square degrees) down to $i_{\text {vega }}=22.2(\approx 27,000)$ were visually classified using the survey's deepest filter, the $i^{\prime}$ band. The apparent magnitude distributions were similar for the D1 and D2 field. There were small differences $(<10 \%)$ at the bright end but were due to the D1 field covering 0.05 square degrees more than D2, coupled with a larger number of bright stars in the D2 field, resulting in a larger masked out region.

Each galaxy was viewed on a computer screen with DS9 (Joye \& Mandel 2003), and the classification was logged through 
keypad entry to a file. More than $90 \%$ of galaxies with apparent magnitudes brighter than $i^{\prime} \leqslant 21.9$ were able to be visually classified. Although some galaxies at these apparent magnitudes had angular sizes too small for a reliable classification to be made, the majority of the remaining $10 \%$ lied within halos of bright stars, or bad regions of the images that were not masked out. At faint magnitudes visual classification becomes increasingly difficult and less reliable, due typically to the smaller angular size of the objects. We therefore imposed an apparent magnitude cutoff of $i_{\text {vega }}^{\prime} \leqslant 21.9$ to ensure highly reliable classifications. Our final morphological catalog consists of 25,194 galaxies.

Galaxies were classified as interacting or merging if they exhibited a tidal tail or tidal bridge between galaxies. These two tidal signatures encompass early through to advanced stage mergers. We then further sub-classified these objects in a simplistic way according to the length of their longest tidal tail as well as the number of tails to see if they were correlated with other properties such as the SFR or active galactic nucleus (AGN) activity. The length of the tidal tail was defined in relation to the angular size of the host galaxy. Figure 1 provides $i^{\prime}$ band and color composite $\left(g^{\prime}, r^{\prime}, i^{\prime}\right)$ examples of galaxies classified as interacting. Galaxy mergers were identified and classified into the following primary categories:

Long tidal tailed galaxies. Galaxies exhibiting a tidal tail longer than the diameter of the host galaxy. These mergers primarily represent intermediate to late stage interactions after a first encounter.

Medium tidal tailed galaxies. These systems have a tidal tail length approximately equal to the diameter of the host galaxy, and also probe interactions after the first passage to advanced mergers.

Short tidal tailed galaxies. This classification identifies galaxies with tidal tails that are less than the diameter of the host galaxy. This classification suffers from the most contamination from spiral arms being misidentified as tidal tails. Therefore, short tidal tailed galaxies are only included in the potentially interacting sample and not considered in the primary analysis of this paper.

Tidally bridged galaxies. Galaxies found to have a tidal bridge linking it to another galaxy. This classification represents a narrower time frame in the merger sequence, typically immediately after the first encounter, or second passage, and is in turn well suited to comparative studies with simulations.

Double nuclei. Our final primary merger classification identifies mainly advanced stage mergers, with two or more nuclei overlapping in a common envelope. Since some galaxies with apparent double nuclei are a result of line-of-sight projections, this classification was further separated into those galaxies with a multiple nuclei and additional evidence of a tidal tail and those with no tidal tail signatures.

Table 2 outlines the classification scheme and the number of galaxies identified in each merger class. Ultimately, we find 1586 galaxies with clear signatures of a tidal tail or bridge, with an additional 970 potentially interacting galaxies.

The sample of potentially interacting galaxies is comprised of galaxies with a double nuclei with no evidence of a tidal tail, which can be a result of projection, and those with "short" tidal tails since these tails are less pronounced and can be confused with spiral arms. However, if the "short" tailed galaxy had secondary evidence, such as the presence of a double nuclei or a tidal bridge, they were considered to be undergoing a merger. Since an aim of this paper was to measure a lower limit of the
Table 2

Classification Scheme for Interacting Galaxies

\begin{tabular}{lr}
\hline \hline \multicolumn{1}{c}{ General Description } & $N$ \\
\hline "Long Tidal Tails": Tail Length > Diameter of Host & 157 \\
\hline Galaxies with a long tidal tail: 1 tail & 183 \\
Galaxies with a long tidal tail: 2 tails & 36 \\
Galaxies with a long tidal tail: > 2 tails & 293 \\
\hline "Medium Tidal Tails": Tail Length Diameter of Host & 138 \\
\hline Galaxies with a medium length tidal tail: 1 tail & 25 \\
Galaxies with a medium length tidal tail: 2 tails & \\
Galaxies with a medium length tidal tail: $>2$ tails & 486 \\
Close Galaxy-Galaxy Pairs with Tidal Bridges & 48 \\
Galaxy in a close pair with bridge, no tail & 49 \\
Galaxy in a close pair with bridge, + short tidal tail & 50 \\
Close Pair with bridge + medium tail(s) & 12 \\
Close Pair with bridge + long tail(s) & 47 \\
\hline Double Nuclei (DN) & 62 \\
\hline Galaxies w/ a DN + short tail(s) & 1586 \\
Galaxies w/ a DN + medium tail(s) & 586 \\
Galaxies w/ a DN + long tail(s) & 384 \\
\hline Total Number of Confidently Interacting Galaxies & \\
\hline Potential Galaxy Interactions & \\
\hline "Short Tidal Tails": Tail Length < Diameter of Host & \\
Galaxies with a double nuclei (DN), no tidal tail & \\
\hline
\end{tabular}

Note. $N$ specifies the number of galaxies in each morphological classification.

fraction of galaxies undergoing a major merger, the sample of potentially interacting galaxies was included only as secondary measure of the merger fraction (see Section 4) and not in the merger rate determination or the general conclusions of this work.

\subsection{A Re-classification Experiment}

A potential bias in using a visual classification technique is its reproducibility when other individuals inspect the same sample of galaxies. A key criteria of this work, however, was the requirement of strong tidal signatures before a galaxy was deemed "interacting," dramatically reducing the classification variance by different individuals. C.B. visually classified all galaxies under study. To cross-check these classifications, M.S. and K. Bundy (KB) classified a sub-sample of 700 objects. This sample of galaxies was randomly selected from within four weighted parent classifications (490 galaxies with tidal tails, 110 galaxies with a tidal bridge, and 50 galaxies classified as potentially interacting, and 50 deemed non-mergers).

This re-classification experiment was a blind study, both K.B. and M.S. were given no information regarding the galaxies they were classifying. The classification was performed in the same manner as described in Section 3.2. Of the galaxies used in the classification experiment, $86 \%$ had been categorized as "interacting" prior to the experiment (by C.B.). Both K.B. and M.S. classified $88 \%$ and $87 \%$ of the galaxies in the experiment to be "interacting." The additional $1 \%-2 \%$ found by K.B. and K.S. to be interacting were predominantly classified as "potential" galaxy interactions with $<0.5 \%$ deemed non-mergers by C.B. The strong agreement between different individuals regarding which galaxies are "interacting" is a result of the robust visual criteria a galaxy must exhibit before it is classed as undergoing an interaction. We are therefore excluding many potentially interacting systems as well as those with tidal features below the 

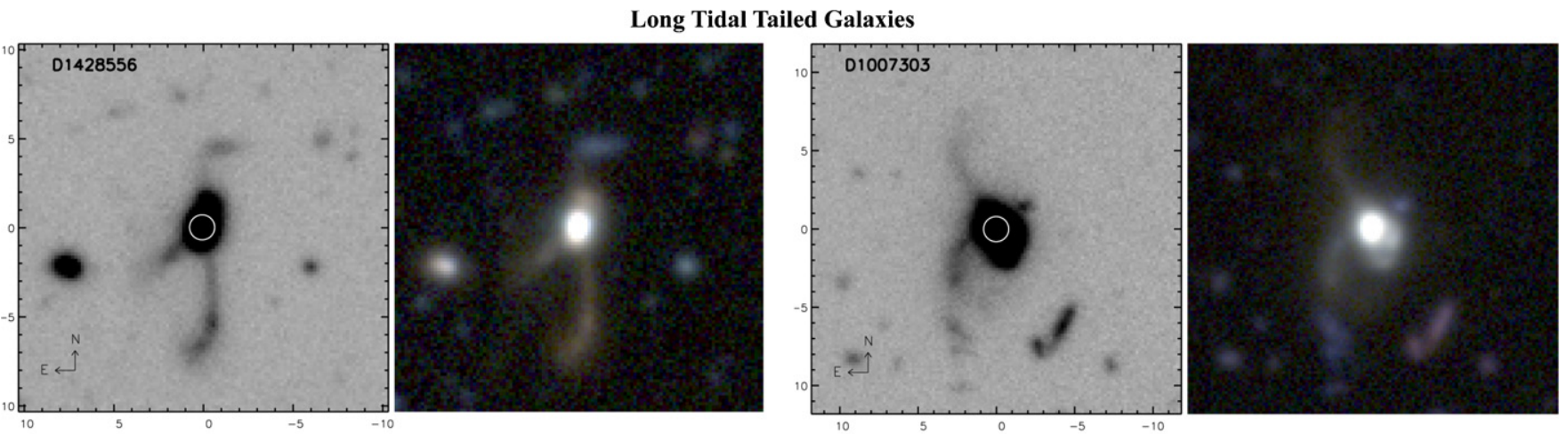

Medium Tidal Tailed Galaxies
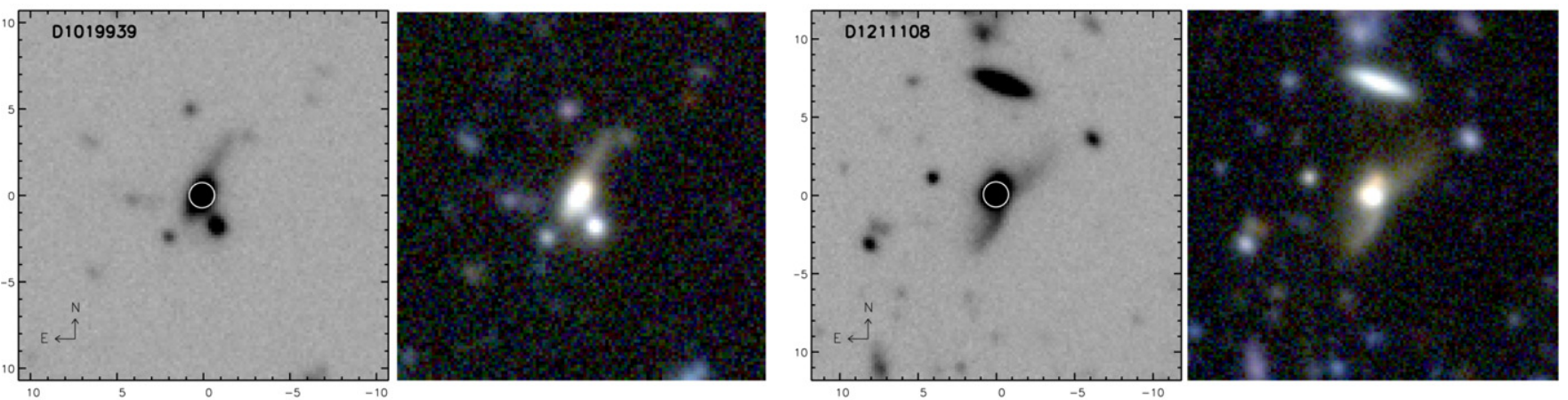

Tidally Bridged Close Pairs
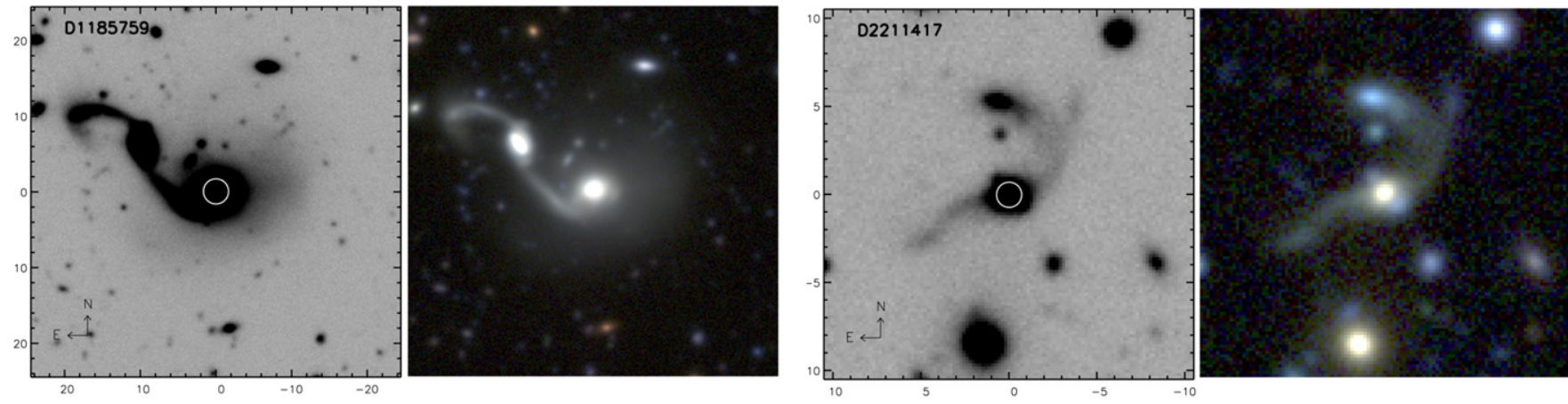

Double Nuclei with Tidal Tail(s)
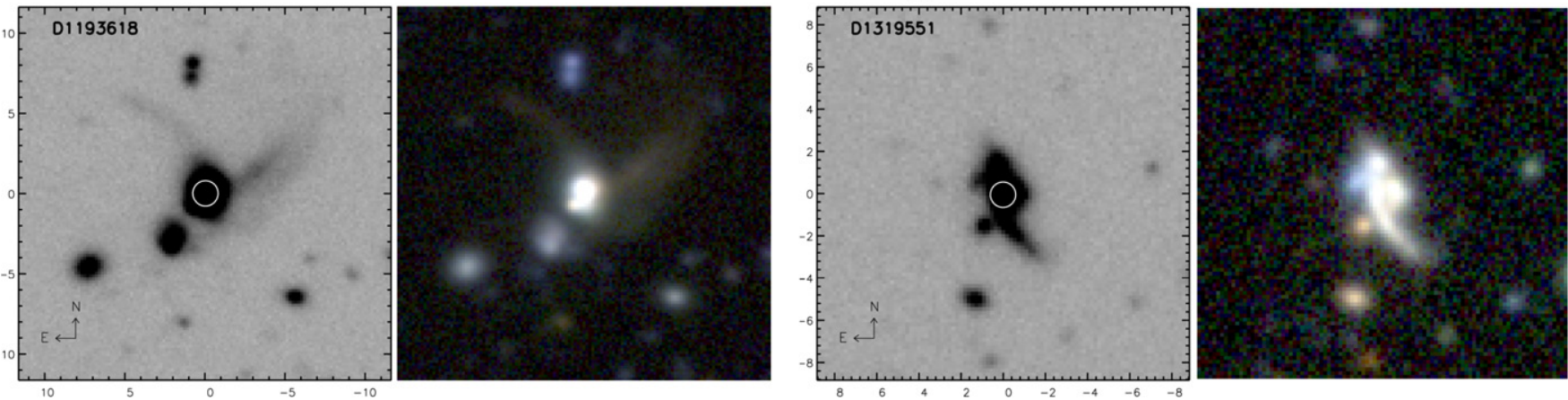

Figure 1. Examples of galaxies classified to have tidal tails or bridges from the CFHTLS-Deep Catalog of Interacting Galaxies. The $i^{\prime}$-band images (left) and color composites $\left(g^{\prime}, r^{\prime}, i^{\prime}\right)$ range in class from galaxies with tidal tails (various lengths), close pairs with tidal bridges to double nuclei with tidal tails. Each stamp is $100 h^{-1}$ $\mathrm{kpc}$ on a side. The white circle marks the galaxy that has been classified. The $X Y$ axes are in arcseconds.

sensitivities of our survey. The results that follow are therefore secure lower limits.

As an additional test, C.B. re-classified a set of 500 galaxies (350 interacting, 150 non-interacting) randomly selected. This blind self-test addresses the reproducibility of the authors own classifications. The classifications remained the same $97 \%$ of the time. The $3 \%$ variation was primarily a result of galaxies being classed into morphologically similar types, for example, an intermediate tidal tailed galaxy being classed as having a short tidal tail, and not into grossly different types. 


\subsection{Classification Completeness}

When investigating how the fraction of interacting galaxies changes from low $-z$ to high- $z$, it is important to investigate how tidal features become less resolved and fainter due to cosmological effects. To address this question, we have artificially redshifted bright nearby galaxies with tidal features in our sample out to higher redshifts. We then re-classify the redshifted galaxies to determine the point in redshift space that the tidal signatures are too weak, and the galaxy would not be classified as interacting.

When simulating an image of a low redshift galaxy to how it would appear at a higher redshift, multiple factors must be considered. First, we rebin the image, ensuring that flux is conserved, to account for the reduction in apparent size of the galaxy when it is viewed at higher redshift. Second, the dependence of surface brightness $(\mathrm{SB})$ with redshift $(\mathrm{SB} \propto$ $\left.(1+z)^{-4}\right)$, as well as $k$-correction effects needs to be accounted for. In order to accurately simulate the appearances of galaxies at high redshift, we carefully selected the lowest redshift galaxies possible with similar luminosities to those probed at the high redshift end of our data $(z \sim 0.7-1.0)$. Our resultant sample consisted of 54 galaxies with tidal features between $0.3 \leqslant z \leqslant 0.45$ and $M_{g} \leqslant-21.0$.

At redshifts between $0.4 \leqslant z \leqslant 0.9$, the $i^{\prime}$ band, which was the filter used for visual classification, is probing rest frame $g^{\prime}$. Therefore, to help account for the $k$-correction the $r^{\prime}$-band images of our sample of low- $z$ galaxies $(0.3 \leqslant z \leqslant 0.45)$ were artificially redshifted, as they also probe rest $g^{\prime}$, like that of the $i^{\prime}$-band images used for classification of the main sample. When redshifting the low $z$ galaxies to our highest redshift bin $(z \sim 1-1.15)$, ideally the $g^{\prime}$-band images should be used so that the same rest wavelengths are being compared in original and redshifted samples. However, the $g^{\prime}$ images were not sufficiently deep, and in turn the $r^{\prime}$-band images were used.

Ultimately, a sample of 54 low redshift galaxies were redshifted out to five different redshifts, $z=0.55,0.70,0.85,1.0$, and 1.15. The galaxies were re-classified at each interval for the presence of tidal features. The recovery rate remains high out to $z \sim 0.85$, where we can still identify strong tidal features in $\sim 80 \%$ of the redshifted galaxies. Throughout the analysis, the recovery rate $(75 \%$ at $z=0.9)$ is referred to as a completeness limit, as it quantifies our survey's sensitivity to tidal features. The rate of recovery could also be used as a correction factor when determining the fraction of galaxy mergers at a given redshift (as discussed in Section 4).

\subsection{Sample Selection}

In order to trace the fraction of merging galaxies with redshift, we first need to define a sample of galaxies that probe the same stellar mass or luminosity ranges over the entire redshift range. In Sections 3.2 and 3.4, we found that visual classifications could be securely made to $i_{\text {vega }} \leqslant 21.9$, with $>75 \%$ completeness out to $z=0.9$.

The majority of merger rate studies to date impose luminosity rather than stellar mass limits on their data sets (excluding Conselice et al. 2003, 2008; Bundy et al. 2009). A potential bias with this selection criteria is the inclusion of interacting galaxies into the sample which prior to the merger event may have been fainter than the luminosity cutoff. This selection bias may lead to higher merger fractions or close pair counts (Bundy et al. 2004; Berrier et al. 2006; Patton \& Atfield 2008). However, stellar mass estimates are less affected by merger

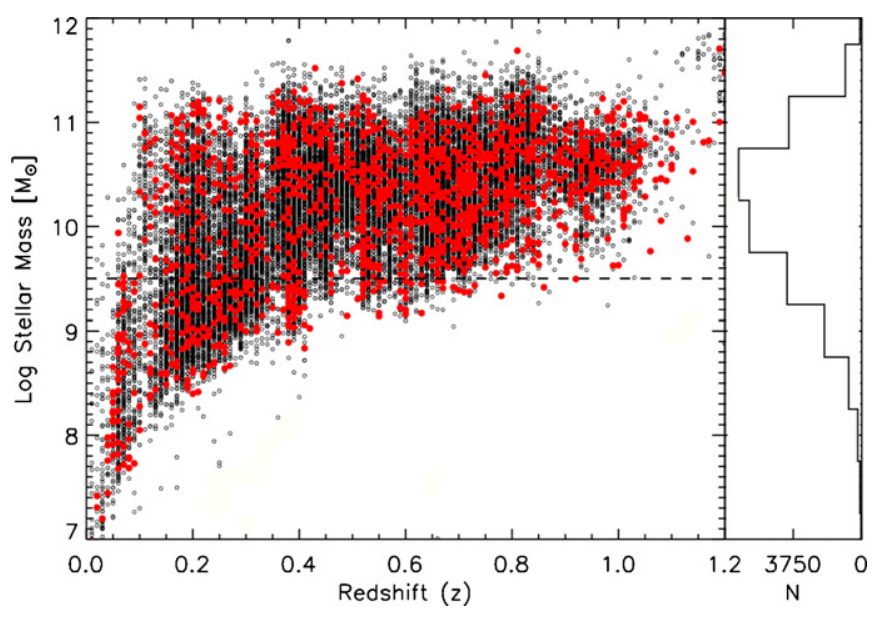

Figure 2. Stellar mass as a function of redshift for the CFHTLS D1 and D2 fields. Galaxies classified as non-interacting are shown as open circles (black) and those undergoing an interaction, filled circles (red). The dashed line defines the stellar mass limit $\left(\log\right.$ mass $\left.>9.5 M_{\odot}\right)$ imposed. The histogram on the right notes the stellar mass distribution of the field.

(A color version of this figure is available in the online journal.)

induced star formation (Conselice 2006), and therefore a less biased selection criteria. In the sections to follow, we explore the galaxy interaction fraction using a stellar mass limit of $\log M_{*}\left(M_{\odot}\right) \geqslant 9.5$ (see Figure 2), however, also consider luminosities limits to fairly compare our findings with previous works. The stellar mass and luminosity limits were chosen by balancing incompleteness with sample size.

To first order, our sample was divided into "interacting" and "non-interacting" galaxies. As outlined in Section 3.1, we define "interacting" or "merging" galaxies as those with tidal tail features, more specifically, with the following visual classifications: medium to long tidal tails, galaxies in a close pair linked with a tidal bridge, and those with a clear double nuclei and a tidal tail. We find a total of 1586 interacting galaxies within the D1 and D2 fields combined.

In order to investigate the properties of interacting and merging galaxies, we also need to establish a comparison sample of non-interacting galaxies. We combine all the "noninteracting" classifications, which are primarily composed of spiral or disk-dominated sources to construct a fair comparison sample. We have identified 22,268 non-interacting galaxies.

We applied a traditional template-fitting technique as described in Section 2.2 to acquire photometric redshift estimates for each of our sources. The redshift distribution for both the interacting and non-interacting populations (Figure 3 ) is found to be comparable. This paper probes the merger fraction over the redshift range $0.1<z<1.2$. The upper redshift limit of 1.2 is due in combination to the lack of sources at redshifts $>1.2$ (caused by the apparent magnitude limit required for accurate visual classification), and the fact that the $4000 \AA$ break begins to move beyond our bluest filter, reducing the accuracy of the photometric redshifts. Ultimately, the CFHTLS-Deep Catalog of Interacting galaxies contains 1240 interacting galaxies between $0.1<z<1.2, i_{\text {vega }}<21.9$, with stellar masses $>10^{9.5} \mathrm{M}_{\odot}$.

\section{THE GALAXY INTERACTION FRACTION (GIF) AT $0.1<z<1.2$}

Tidal tails and bridges are a result of gravitational encounters between two or more galaxies (e.g., Toomre \& Toomre 1972), and are nearly foolproof signatures of a recent or ongoing 


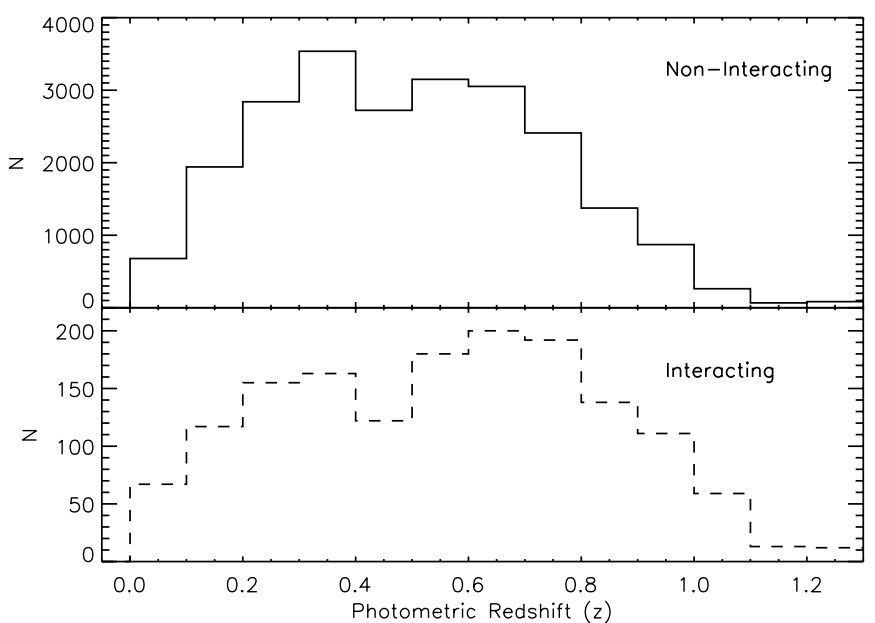

Figure 3. Redshift distribution for all galaxies classified as non-interacting (upper) and interacting (lower, dashed). The mean photometric redshifts for the two samples are 0.51 (non-interacting) and 0.58 (interacting). The two samples have comparable redshift distributions.

galaxy interaction. The statistics of galaxies with extended tidal features (tidal bridges and tails) is a powerful tool to study the evolution of the galaxy interaction fraction (GIF). In this section, we present our analysis of the merger fraction between $0.1<z<1.2$ using the CFHTLS Deep Catalog of Interacting Galaxies. This catalog is the largest sample of interacting galaxies in the literature over this redshift range.

The number of galaxies exhibiting strong tidally induced structures like tails and bridges is a relatively simple and robust, lower limit measure of the interaction fraction. CFHTLS-Deep is sensitive to tidal features with surface brightnesses down to $i^{\prime} \lesssim 29 \mathrm{mag} \operatorname{arcsecond}^{-2}$. Our approach is simply to compare the number of galaxies with tidal features $\left(N_{\text {Int }}\right)$ to the total number of galaxies within the same absolute magnitude or stellar mass ranges $\left(N_{\text {Total }}\right)$, as a function of redshift (see Equation (1)). In the case where a pair of galaxies are connected by a tidal bridge, each galaxy will be counted as interacting $\left(N_{\text {Int }}=2\right)$, while a galaxy with a tidal tail and no bridge linking it to another object is counted as a single interaction $\left(N_{\text {Int }}=1\right)$.

$$
\text { Galaxy Interaction Fraction(GIF) }=\frac{N_{\text {int }}}{N_{\text {Total }}}
$$

In Figure 4, we plot the fraction of galaxies with tidal tails, as a function of redshift, assuming $i^{\prime}<21.9$, and stellar masses $>10^{9.5} M_{\odot}$. As previously described in Section 3.2, the interaction classes included in this measurement are "medium" and "long" tidal tailed galaxies, those in a close galaxy pair with a tidal bridge, and those galaxies with a double nuclei and addition morphological evidence of an interaction such as a tidal or bridge.

We find that the fraction of galaxies involved in a merger rises with redshift. Meaning, more galaxies were undergoing a tidal interaction when the universe was about half its current age. At low redshift $(z \sim 0.3)$, the GIF was found to be $\sim 4 \% \pm 0.3 \%$, and nearly triples by $z \sim 0.95$ to $11 \% \pm 0.9 \%$, (see Table 3 ). We characterize the evolution of the galaxy interaction fraction by fitting a simple power-law increase with redshift of the form $\mathrm{GIF}=\mathrm{GIF}_{o}(1+z)^{m}$, where $\mathrm{GIF}_{o}$ is the present day interaction fraction, and $m$ is the power-law index. When all redshift bins in our sample are included $(0.1 \leqslant z \leqslant 1.15)$ we find a best fit $m$ of $2.25 \pm 0.24$ and $\mathrm{GIF}_{0}$ of $2.15 \% \pm 0.25 \%$, weighted by the GIF error bars in each redshift bin.

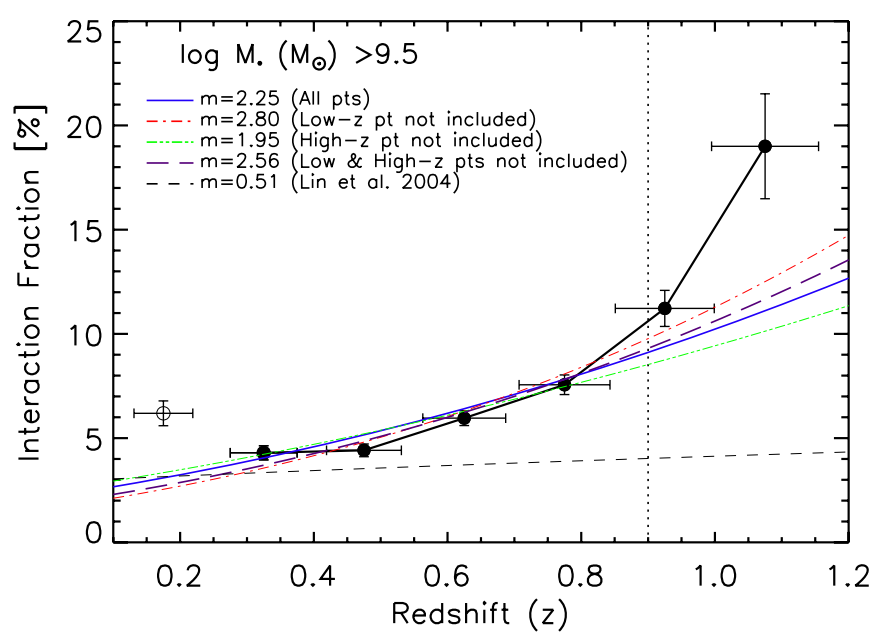

Figure 4. Mass limited galaxy interaction fraction as a function of redshift from CFHTLS-Deep (circles). The colored lines represent the best $(1+z)^{m}$ fits when various data points are included in the analysis. When all points between $0.1 \leqslant z \leqslant 1.15$ (with stellar masses $\geqslant 10^{9.5} M_{\odot}$ ) are included the best fit of $(1+z)^{m}$ is $m=2.25 \pm 0.24$ (blue, solid). When the lowest redshift point is not included, $m=2.8 \pm 0.25$ (red, dash-dot), only the high- $z$ point removed, $m=1.95 \pm 0.2$ (green, dash-dot-dot), and when both the low and high- $z$ points are not included $m=2.56 \pm 0.24$ (purple, long dash). The black dashed line shows the result of Lin et al. (2004); Lotz et al. (2008b) $(m=0.51 \pm 0.28)$ who find mild to no evolution in the close pair fraction. The error bars are derived using Poisson statistics, while the horizontal errors come from the uncertainty in the photometric redshift. The vertical dotted line represents the $75 \%$ completeness limit. The open circle notes the data point suffering from the selection bias discussed in Section 4.1.

(A color version of this figure is available in the online journal.)

Table 3

Inferred Galaxy Interaction Statistics

\begin{tabular}{cccc}
\hline \hline Redshift & $N_{\text {INT }}$ & $N_{\text {Total }}$ & Interaction Fraction [\%] \\
\hline $0.10-0.25$ & 108 & 1745 & $6.2 \pm 0.6$ \\
$0.25-0.40$ & 160 & 3725 & $4.3 \pm 0.3$ \\
$0.40-0.55$ & 209 & 4736 & $4.4 \pm 0.3$ \\
$0.55-0.70$ & 280 & 4700 & $6.0 \pm 0.4$ \\
$0.70-0.85$ & 258 & 3413 & $7.6 \pm 0.5$ \\
$0.85-1.00$ & 168 & 1497 & $11.2 \pm 0.9$ \\
$1.00-1.15$ & 57 & 300 & $19.0 \pm 2.5$ \\
\hline
\end{tabular}

Notes. $N_{\text {Int }}$ specifies the number of interacting galaxies. $N_{\text {Total }}$ is the overall number of galaxies in the sample. Errors are Poisson statistics.

As discussed in the following section there may be some potential biases at the low and high redshift ends of our analysis. Therefore, the evolution of the GIF was also fit using various redshift ranges as shown in Figure 4. If the lowest redshift bin (0.1-0.25) is excluded, a best-fit power-law index of $m=2.80 \pm 0.25$ is derived. If the highest redshift bin 1.0-1.15 (the least confident) is excluded $m=1.95 \pm 0.25$, the lowest degree of evolution found in our analysis. Finally, if both the lowest and highest redshift bins are removed, a value for $m$ of $2.56 \pm 0.24$ is found. It is clear that even assuming the minimum value of $m$ found in this analysis, a mild to nonevolving merger fraction $(m<1.5)$ with redshift is ruled out at the $\gtrsim 4 \sigma$ confidence level.

\subsection{Potential Biases}

In many previous merger fraction studies, any evolution measured in the merger fraction with redshift typically depends strongly on the either the lowest redshift $(z \lesssim 0.2)$ data point where volume effects come into play, or the highest most incom- 


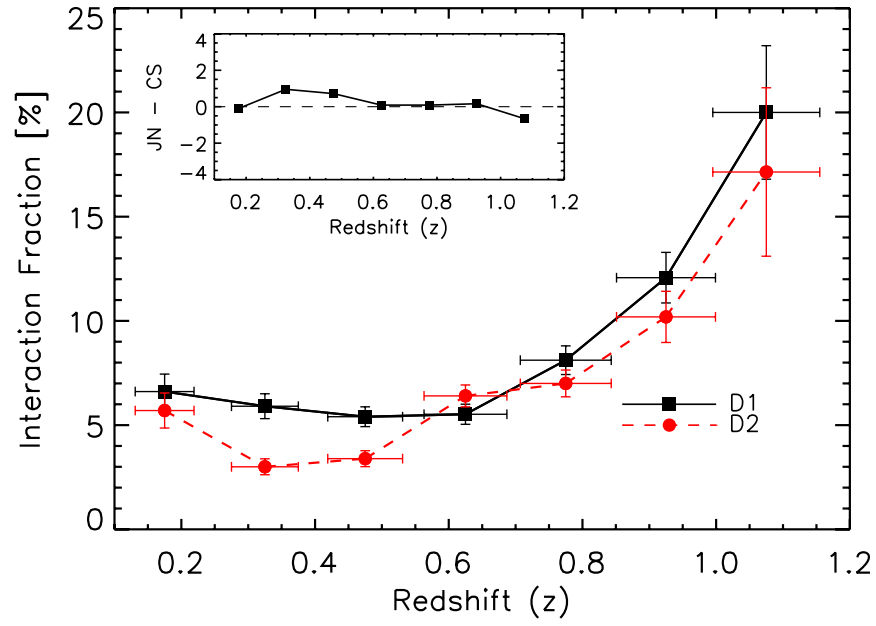

Figure 5. Galaxy interaction fraction as a function of redshift from CFHTLSDeep for the D1 (filled squares, black) and the D2 field (filled circles, red). Cosmic variance dominates the errors of the GIF at $z<0.5$; however, at higher redshift the Poisson errors are larger. Inset panel shows the difference between Jackknife (JN) and counting statistic (CS) errors.

(A color version of this figure is available in the online journal.)

plete redshift bin. Although like many other works our lowest and highest redshift bins suffer biases or incompleteness, we still find evolution of the merger fraction of at least $m \sim 2$ over our most secure and complete redshift range $(0.3<z<0.9)$.

The interaction fraction for the lowest redshift bin $(0.1 \geqslant z<$ 0.25 ) is elevated compared to the GIF found between $0.25 \leqslant$ $z \leqslant 0.6$. This is likely related to two factors. The first is the larger angular size of low redshift galaxies making tidal features more easily detected. The second and likely more dominant factor is that the lower redshift bins are more susceptible to cosmic variance as shown in Figure 5 and discussed further in Section 4.2.

Another potential bias to consider is that as we probe higher redshifts, $z>0.9$, we begin to more closely observe the UV ( $u^{*}$-band) which is dominated by massive young $\mathrm{O}$ and $\mathrm{B}$ stars. Therefore, star formation in tidal tails and bridges may be more easily visible resulting in a higher GIF at larger redshifts. Since the $u^{*}$-band images are not a comparable depth to the $i^{\prime}$-band data, a simulation similar to that outlined in Section 3.4 was run on the sub-sample of lower redshift tidal tailed galaxies but with a 1 mag enhancement in surface brightness. The resultant GIF increased by $\lesssim 1 \%$ at $z=1$. It is therefore unlikely that this potential bias is solely or even largely responsible for the higher GIF at $z \sim 1$, especially considering that the completeness test estimates that we are missing a much larger fraction, $15 \%-30 \%$ of tidal tailed galaxies, at $z \sim 1$ due to cosmological dimming effects. Despite these potential biases, our analysis suggests that the evolution in the GIF is a real physical effect, meaning their were more galaxies undergoing mergers at earlier times out to $z \sim 1$.

\subsection{GIF: Cosmic Variance}

A benefit of CFHTLS-Deep is that it is spread over multiple fields allowing for the study of how cosmic variance can affect measurements. In Figure 5, the GIF is presented separately for the D1 and D2 fields. Both fields show an evolving GIF with redshift; however, the steepness of the evolution varies, stressing the importance of studying multiple large fields. The jackknife errors, representing the impact of cosmic variance, were calculated by considering the difference in the GIF between the D1 and D2 fields (see Figure 5 inset). At lower redshifts, where the volume being probed is less, we find the GIF is affected more strongly by cosmic variance, while at $z>0.6$ little variation in the GIF is seen between the fields.

\subsection{GIF: Comparison with Previous Works}

A key consideration when attempting to compare results from previous merger rate studies is the similarity of the objects being probed, i.e., comparable luminosity, or stellar mass limits as well as the selection technique. Numerical results of the merger fraction using morphology should not be compared directly to a close galaxy pair fraction, since merger selection techniques have very different observability timescales. Direct comparisons, however, of the galaxy merger rate derived with different selection techniques can be made since the close pair or merger fractions are normalized by the appropriate timescale. In Section 5.1, we compare our merger rate with those of previous studies. It must be noted that the merger timescale can vary widely depending on the initial merger parameters and remains a large source of uncertainty. Therefore, a comparison of merger fractions is warranted, since they are proportional to the evolution of the merger rate.

In this section, we compare our results of the merger fraction and its evolution with redshift with other morphologically based works. Since we use a morphological approach in identifying interactions a first-order comparison would be studies that also consider the visual appearance of a galaxy whether it be qualitative or quantitative, like those of Le Fèvre et al. (2000) and Conselice et al. (2003) to name a couple. Figure 6 compares our work with other morphologically based estimates of the merger fraction. The only other study which limited their sample by stellar mass rather than luminosity in this redshift range is Conselice et al. (2003). At intermediate redshifts, we find a merger fraction of $6 \% \pm 0.4 \%$, in good agreement with Conselice et al. (2003) who find $7 \% \pm 5 \%$, and consistent results at $z \sim 1$. Reasonable comparisons can be made with other studies since we are also probing similar luminosity ranges (see Section 4.5). A major obstacle that has plagued merger rate studies is small sample sizes inspecting only a few tens to a hundred galaxies, resulting in large $(5 \%-15 \%)$ uncertainties making it difficult to confidently measure both the merger fraction and its evolution with redshift. In this study, we inspected more than 25,000 galaxies, a factor of $\gtrsim 10$ more than previous morphological investigations. We find that within the uncertainty most of the studies shown in Figure 6 are consistent with our GIF measurements.

This work has presented a statistically secure lower limit of the interaction fraction for galaxies with stellar masses $M_{*}>10^{9.5} M_{\odot}$ as a function of redshift. The fraction of galaxies undergoing tidal interactions is surely higher than the rate reported here, as this study is limited to higher surface brightness features, and only included the most confident tidal features in its analysis.

In Figure 6, we recalculated the GIF applying the completeness correction estimated in Section 3.4. Recall that this correction factor accounts for the number of mergers not identified as a function of redshift due to cosmological effects. Evolution of the galaxy merger fraction is strikingly apparent. We find that the best fit of $\left(\mathrm{GIF}_{0}, m\right)$ is $(0.015 \pm 0.002,3.31 \pm 0.22)$. Also shown in Figure 6 is the GIF with the inclusion of less confident signatures of tidal interactions or mergers, such as galaxies with 


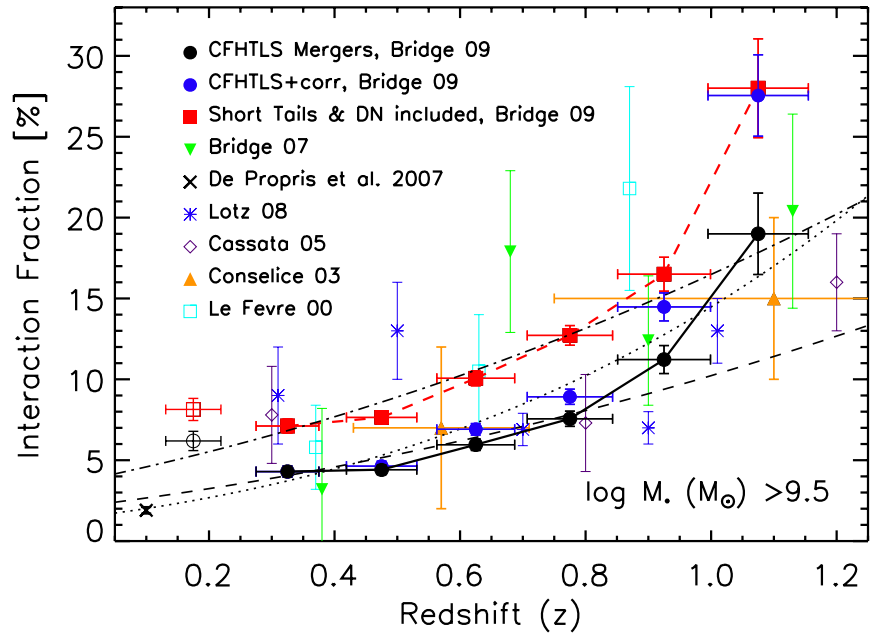

Figure 6. Plot of the mass limited GIF as a function of redshift from CFHTLSDeep using the primary interacting sample (filled circles, black) and the secondary sample (squares, red) that include the primary merger sample and galaxies with "short" tidal tails, and double nuclei. Blue circles highlight the interaction fraction corrected for completeness. Observations of the merger fraction are complied from Lotz et al. (2008b, blue stars), Bridge et al. (2007, green, inverted triangles), De Propris et al. (2007, black x), Cassata et al. (2005, purple diamonds), Conselice et al. (2003, orange, triangles), and Le Fèvre et al. (2000, cyan, open squares). The black lines outline the best fit of form $(1+z)^{m}$ for the various samples. $m=2.25 \pm 0.24$ for the primary merger sample (dashed), while the GIF corrected for completeness (dotted line) has a stepper fit of $m=3.31 \pm 0.18$. The best fit for the primary sample with the inclusion of probable interactions is $m=2.14 \pm 0.17$ (dash-dotted). All fits include the lowest redshift points of this study (open circle/square) which suffer from a selection bias (Section 4.1), strengthening $m=2.25$ as a lower limit. It is clear that a non-evolving merger fraction is inconsistent with the CFHTLS-Deep data. (A color version of this figure is available in the online journal.)

"short" tidal tails (tail length less than diameter of host), and galaxies with a double nuclei and no secondary signs of an interaction. This resulted in an additional 767 interacting galaxies being included in the analysis. The resultant GIF is on average $\sim 5 \%$ higher than our lower limit, and its evolution with redshift ( $m=2.14 \pm 0.17$, compared to $m=2.25 \pm 0.24$ ) traces the lower limit GIF very well over the full redshift range. Although these classifications were deemed to be less confident, they are likely dominated by galaxies which are truly merging as there is reasonable agreement with the completeness corrected GIF at high redshift.

Overall we find that between $0.1<z<1.2$, our value of $m(m=2.25)$ is in good agreement with $m=2.2 \pm 0.3$ found by Cassata et al. (2005), $m=2.12$ reported by Bridge et al. (2007) and Conselice et al. (2003). The merger fraction accounting for cosmological is in better agreement with Le Fèvre et al. (2000) which find $m=3.4 \pm 0.6$. Our results do differ, however, from Lotz et al. (2008b) $(m<1)$ which utilized the DEEP2 survey. There are some factors that may explain the discrepancy aside from the choice of luminosity versus stellar mass. First, the $G-M_{20}$ structural parameters have a significantly short observability timescale $(0.2 \mathrm{Gyr})$ compared to our visual classification $(0.8 \mathrm{Gyr})$, which could account for the lower merger fractions found at some redshifts. Second, mergers were identified using the Gini $-M_{20}$ region calibrated by the location of low redshift ULIRGs on this diagram (Lotz et al. 2004). Kampczyk et al. (2007) found that only $\sim 6 \%$ of visually classified mergers lie in the region of the Gini $-M_{20}$ used by Lotz et al. (2008b). Lotz et al. (2008a) have also found through simulations that when a merger has a single nucleus,

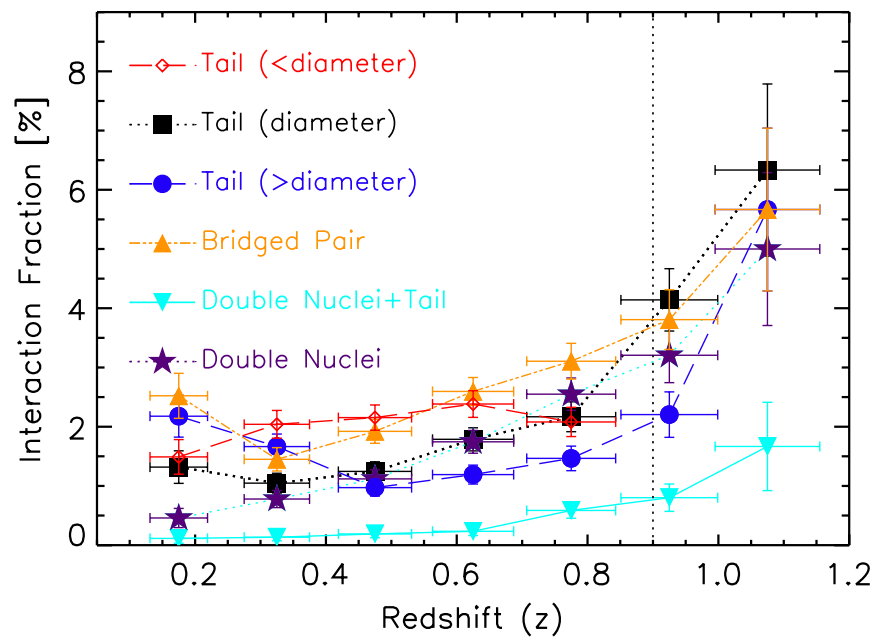

Figure 7. Galaxy interaction fraction as a function of redshift for different interaction classes. Short tidal tails are shown in diamonds (red), medium tails squares (black), long tails circles (blue), bridged close pairs upward triangle (orange), galaxies with a double nuclei and a tidal tail downward triangle (green), and double nuclei with no tail star (violet). The error bars are derived using Poisson statistics, while the horizontal errors come from the uncertainty in the photometric redshift. The vertical dotted line represents the $75 \%$ completeness limit. A stellar mass limit of $10^{9.5}\left(M_{\odot}\right)$ has been imposed.

(A color version of this figure is available in the online journal.)

the Gini $-M_{20}$ merger classification has a detection efficiency of $46 \%$. Third, the mild merger evolution reported by Lotz et al. (2008b) is strongly tied to the two lowest redshift bins which suffer from the largest errors, while the merger fractions with the smallest uncertainty are in better agreement with our findings. Finally, cosmic variance may also have an impact, as Lotz et al. (2008b) uses a single field on the sky.

As discussed earlier, even assuming the minimum value of $m$ found in this analysis, a mild to non-evolving merger fraction $(m<1.5)$ is ruled out at the $\gtrsim 4 \sigma$ confidence level.

\subsection{GIF: Interaction Classes}

The large area of CFHTLS-Deep allows us to further explore the fraction of galaxies interacting at various stages of the merger process and how they evolve with redshift. Figure 7 illustrates the GIF for the different merger classifications. The sample was again restricted to be brighter than $i^{\prime} \leqslant 21.9$ to ensure confident classifications and to have stellar masses $\log M_{*}\left(M_{\odot}\right) \geqslant 9.5$ to probe similar mass galaxies at low and high redshifts. We find that all interacting galaxy classes show at least some evidence of evolution with redshift.

The specific values of the interaction fractions for each merger class most likely vary from one another for two reasons. First, each classification represents a snapshot of a stage of the merger process, and various signatures have longer durations. For example, a galaxy with a tidal bridge can be identified at any point after the first encounter, and until the nuclei coalesce, while a galaxy with a visible double nuclei and a tidal tail may be visible for a shorter timescale as the tidal tail may have faded below the SB limit resulting in this classification found less frequently. A second factor relates to the resolution of the images, as ground-based imaging of very late stage mergers, with close double nuclei may be blurred into a single nucleus again reducing the number of late stage mergers identified. 


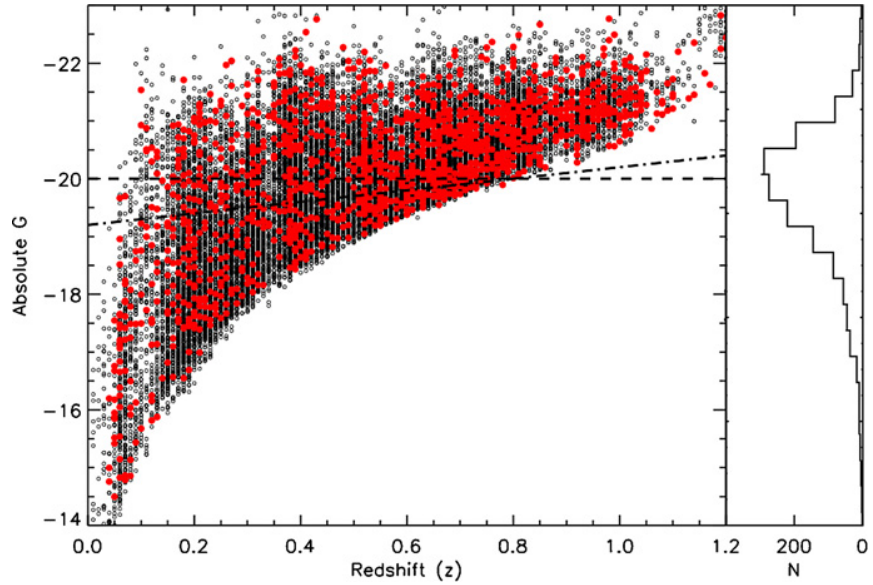

Figure 8. Absolute $g^{\prime}$-band luminosity as a function of redshift for galaxies classified as non-interacting (open circles, black) and those undergoing an interaction (filled circles, red). The dashed lines define the lower absolute magnitude limits considered in the following analysis section; (dashed) no luminosity evolution, (dashed-dotted) includes luminosity evolution $Q=1$. The histogram on the right shows the absolute $g^{\prime}$ magnitude distribution for the interacting galaxy sample.

(A color version of this figure is available in the online journal.)

\subsection{Interaction Fraction: Luminosity Dependence}

After estimating a lower limit for the galaxy interaction fraction, we now explore the sensitivity of the results to various galaxy properties. Many previous merger rate studies have found the merger rate to depend on optical luminosity (Patton et al. 1997, 2000; Conselice et al. 2003; Xu et al. 2004). In the following sections, we apply various optical luminosity limits to our sample and re-analyze the GIF to explore any possible luminosity dependence. Figure 8 shows the absolute $g^{\prime}$-band magnitudes $\left(M_{G}\right)$ for the interacting (red) and non-interacting (black) populations in the combined D1 and D2 fields. It also outlines the $M_{G}$ limits imposed to aid in a more complete comparison of galaxies at high and low redshifts with and without luminosity evolution.

We chose a minimum $\left(M_{\min }\right)$ luminosity limit, $M_{G} \leqslant-20$, which is a few tenths of a magnitude brighter than $M_{G}^{*}$ to balance the completeness at high redshift with probing $M_{G}^{*}$ as closely as possible. The sample was divided into "bright" $\left(M_{G}<-21.0\right)$ and "faint" $\left(-21.0 \leqslant M_{G} \leqslant-20.0\right)$ galaxies to study the impact optical luminosity may have on the frequency of observed galaxy interactions. Figure 9 presents the GIF as a function of redshift for the two luminosity ranges. A clear dependence of the GIF on $M_{G}$ is evident out to $z \sim 0.7$, after which bright (blue points in Figure 9) and faint (red points) galaxies have statistically similar interaction fractions. Within the optical luminosities outlined above, more luminous galaxies show tidal signatures more frequently than less luminous galaxies. It must be considered, however, that this result may be either entirely or in part due to the ease of identifying tidal tails in brighter galaxies, rather than a true increase in the frequency of interactions in more luminous galaxies. The level of this effect is difficult to quantify and requires deeper images of these lower luminosity galaxies to see if tidal features are evident.

Luminous galaxies below $z<0.6$ have interaction fractions up to 2 times higher than the sample of lower luminosity galaxies. Overall, we find that between $0.2 \leqslant z \leqslant 0.7$ the GIF for more luminous galaxies remains fairly constant, while the GIF for fainter galaxies shows mild evolution in this redshift
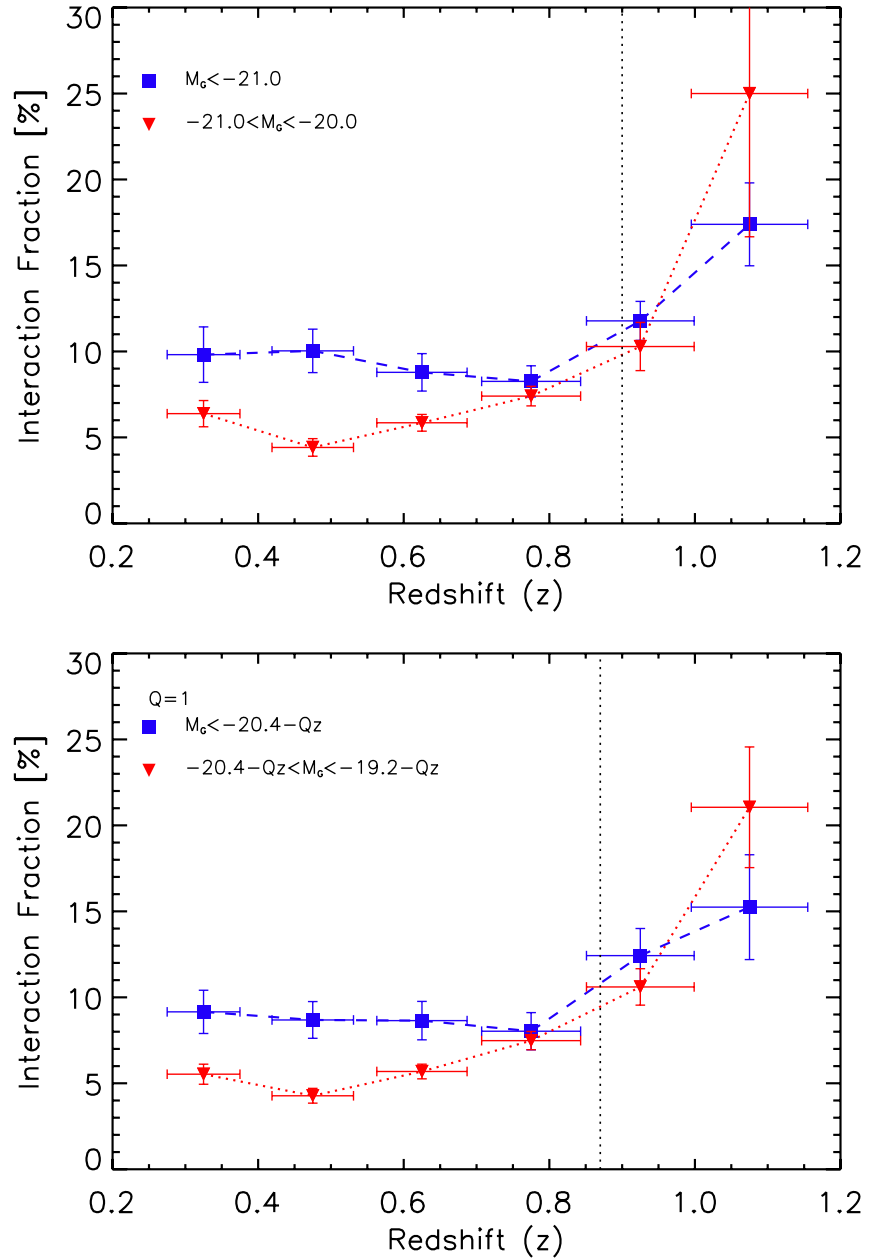

Figure 9. Galaxy interaction fraction as a function of redshift, assuming no luminosity evolution, $Q=0$ (top) and $Q=1$ (bottom). The GIF is not strongly dependent on the assumption of luminosity evolution. At lower redshift $(z<0.6)$, more luminous galaxies (squares, blue) have a GIF up to 2 times that of lower luminosity galaxies (downward triangle, blue). The error bars are derived using Poisson statistics, while the horizontal errors come from the uncertainty in the photometric redshift. The vertical dotted line represents the $75 \%$ completeness limit.

(A color version of this figure is available in the online journal.)

range. There is evidence of an increasing GIF at $z>0.8$, suggesting that interactions and merging may play a larger role in galaxy evolution at higher redshifts. Although this redshift bin contains $\sim 60$ mergers, caution should be taken with its interpretation as it does suffer from incompleteness, particularly in the lower luminosity sample. At this point, we have assumed that galaxies in the past and those locally have similar optical luminosities. We now proceed with a scenario that includes luminosity evolution.

\subsection{Interaction Fraction: Luminosity Evolution}

When a static luminosity limit is considered for a range of redshifts, one is essentially assuming that the mass-to-light ratio $(M / L)$ of galaxies is the same over that redshift range. In this section, we explore the impact that luminosity evolution may have on the GIF measurement.

There is considerable controversy in the literature as to how galaxies evolve at $z<1$. Although it is agreed that evolution does occur (Lin et al. 1999; Carlberg et al. 2001), at what magnitude is under debate. At the very least galaxies will evolve passively as their stellar populations age, resulting in a gradual 
Table 4

Galaxy Interaction Statistics: Mass Dependence

\begin{tabular}{lcrrc}
\hline \hline $\log$ Stellar Mass $\left(M_{\odot}\right)$ & Redshift & $N_{\text {Total }}$ & $N_{\text {Int }}$ & Interaction Fraction [\%] \\
\hline $9.0 \leqslant M<9.5$ & $0.10-0.30$ & 1611 & 67 & $4.2 \pm 0.5$ \\
& $0.30-0.40$ & 727 & 39 & $5.4 \pm 0.9$ \\
\hline $9.5 \leqslant M<10.0$ & $0.20-0.40$ & 1809 & 47 & $2.6 \pm 0.4$ \\
& $0.40-0.60$ & 1564 & 67 & $4.3 \pm 0.5$ \\
& $0.60-0.80$ & 1216 & 90 & $7.4 \pm 0.8$ \\
& $0.80-1.00$ & 180 & 24 & $13.3 \pm 2.7$ \\
\hline $10.0 \leqslant M$ & $0.20-0.40$ & 2012 & 83 & $4.1 \pm 0.5$ \\
& $0.40-0.60$ & 3205 & 128 & $4.0 \pm 0.4$ \\
& $0.60-0.80$ & 3107 & 207 & $6.7 \pm 0.5$ \\
$10.7 \leqslant M$ & $0.80-1.00$ & 1287 & 124 & $9.6 \pm 0.9$ \\
& $0.40-0.60$ & 1137 & 80 & $7.0 \pm 0.5$ \\
& $0.60-0.80$ & 1528 & 86 & $5.6 \pm 0.6$ \\
& $0.80-1.00$ & 1060 & 102 & $9.6 \pm 1.0$
\end{tabular}

Notes. $N_{\text {Total }}$ is the number of galaxies within the specified stellar mass and redshift range. $N_{\text {Int }}$ the is the number of galaxies confidently classified as interacting within a given redshift and stellar mass range. The errors for the interaction fraction were derived using counting statistics.

fading of their optical light. Hence, at higher redshifts when the galaxies are younger we would expect them to have higher mean luminosities. Detailed luminosity function studies (Lin et al. 1999) showed that a luminosity correction can be applied to a galaxy at redshift $z$, using the expression, $Q z$, where $Q$ is typically taken to be equal to 1 (Lin et al. 1999; Patton et al. 2002).

Luminosity evolution has been considered in some close pair studies. Patton et al. (2002) and Lin et al. (2004) found that $N_{c}$ (the number of companions per galaxy) can be significantly affected by the inclusion or exclusion of luminosity evolution. When luminosity evolution is corrected for, fainter galaxies at lower redshifts are included, resulting in higher pair statistics, and in turn less evolution of the pair fraction with redshift.

To explore the effect luminosity evolution may have on the interaction fraction, we have adopted $Q=1$ and repeated the GIF analysis described in the preceding section, when $Q$ was assumed to be zero. We find a $<1 \%$ difference in the GIF with and without the inclusion of luminosity evolution. Unlike close galaxy pair's studies which typically find an increase in the number of pairs at lower redshift when luminosity evolution is applied, we find a slight decrease $(<1 \%)$ in the GIF, with $Q=1$. This could merely be caused by the increased difficulty in identifying tidal tails in fainter galaxies, or perhaps there is an intrinsic dependence of the GIF on optical luminosity as implied by the "bright" and "faint" sub-samples.

In contrast to Lin et al. (2004) who found a factor of 4 difference in $m$ with $Q=0$ to $Q=1$, we find no significant deviation in the evolution of the GIF with or without a luminosity evolution correction (Figure 9). This discrepancy may be an effect of the different selection techniques. As you probe further down the luminosity function, the number of galaxies increases per unit volume, which could in turn increase the contamination from projection effects and unbound pairs. With the tidal tail methodology, probing fainter magnitudes makes the identification of mergers more challenging. However, the intrinsic close pair fraction or merger fraction may also be a function of luminosity. Measuring the pair or merger fraction as the function of luminosity is highly problematic due to the numerous selection biases and contamination effects. Disentangling these effects is challenging, which is why we have chosen to focus this study on a mass-selected sample rather than a luminosity one.

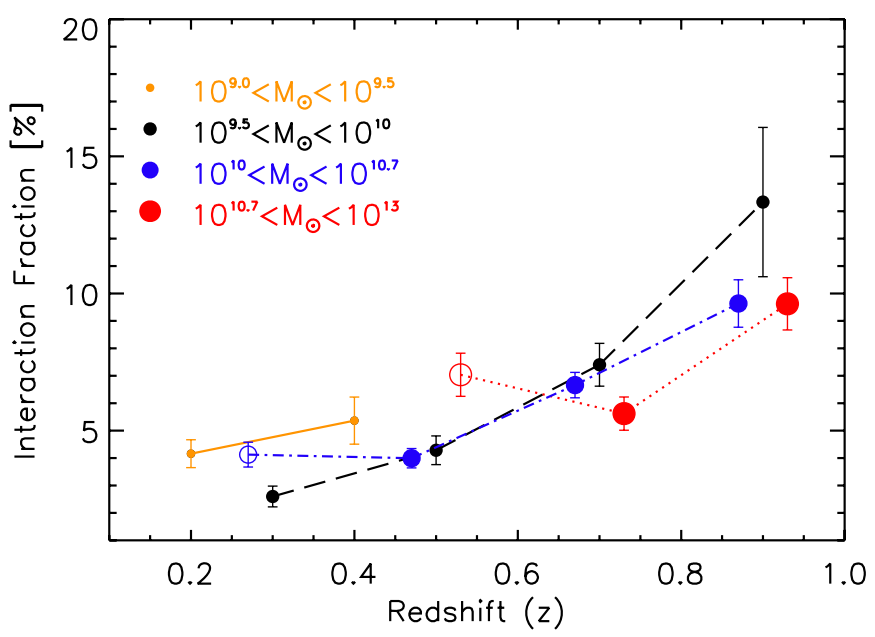

Figure 10. Galaxy interaction fraction as a function of stellar mass and redshift. The data points report the GIF for four stellar mass ranges from $\log M=9.0$ to 13 , and increase in size with stellar mass. The open data points likely suffer from the identification bias discussed in Section 4. There is similar evolution of the GIF for all mass ranges. There is an indication that lower mass galaxies at $z<1$ interact more frequently than high-mass galaxies. This trend is a possible mechanism for downsizing.

(A color version of this figure is available in the online journal.)

\subsection{GIF: Mass Dependence}

Probing the merger fraction as a function of stellar mass provides insight into how galaxies build up over time. In hierarchical assembly, the leading theory for structure formation in the universe, large structures form from the merging of smaller structures. This implies that the most massive galaxies form latest in the history of the universe. Alternative theories postulate that massive galaxies can form through rapid collapse, over short timescales in the early universe. In this section, we aim to address the merger histories of low $\left(M_{*}=10^{9}-10^{10} M_{\odot}\right)$ and high $\left(M_{*}>10^{10.7} M_{\odot}\right)$ mass galaxies and the implications this has on driving galaxy evolution.

Using the five-band optical photometry in the CFHTLS, we estimated the stellar masses of our sources through template fitting (see Section 2.2 for details). Figure 2 details the stellar mass distribution as a function of redshift for our $i^{\prime} \leqslant 21.9$ sample of classified galaxies. Previous merger rate studies derived from observations and theoretical models have suggested that the merger fraction not only varies with optical luminosity as confirmed in the previous section but also depends on stellar mass. It has been suggested that brighter, massive galaxies have the highest merger fractions at $z>1$ (Conselice et al. 2003; Conselice 2006; Maller et al. 2006). In order to investigate how the GIF is affected by stellar mass, we divided our $i^{\prime} \leqslant 21.9$ sample into four mass ranges; low masses $\left(10^{9.0} \leqslant M_{*}<10^{9.5}\right.$ and $\left.10^{9.5} \leqslant M_{*}<10^{10}\right)$, intermediate mass $\left(10^{10} \leqslant M_{*}<10^{10.7}\right)$, and high-mass galaxies $\left(M_{*} \geqslant 10^{10.7}\right)$. The interaction fraction is calculated for each stellar mass range. The GIF statistics are described in Table 4 and plotted in Figure 10. All stellar mass ranges imply a similar evolving GIF with redshift. It should be mentioned that the elevated merger fraction at $z<0.6$ for massive galaxies and $z<0.4$ for intermediate mass galaxies (open circles in Figure 10) is likely the same selection bias noted in Section 4.1.

A key result of Figure 10 is the indication that lower mass galaxies $\left(M_{*} \sim 10^{9}-10^{10} M_{\odot}\right)$ have a higher merger fraction (by $\sim 2 \%-5 \%$ ), compared to more massive galaxies 
$\left(M_{*}>10^{10.7} M_{\odot}\right)$ at $z \lesssim 1$. Since previous studies at higher redshifts $(z>1.5-3)$ have reported the opposite trend, finding the merger fraction of lower mass galaxies less than that found in more massive systems (Conselice et al. 2008) our result suggests (with moderate significance) a transition of galaxy assembly between $z \sim 1$ and 1.5 (i.e., galaxy assembly downsizing). An additional implication of our findings is a potential mechanism for interpreting cosmic downsizing. As mergers are known to trigger star formation (Mihos \& Hernquist 1996; Barton et al. 2000; and Section 6 of this work), a higher merger fraction in lower mass galaxies would aid in the transition of the dominant cites for star formation moving to lower mass systems at lower redshifts.

This trend of a higher galaxy merger fraction in lower mass galaxies at $z<1$ is in contrast with the recent close pair study in the GOODS fields of Bundy et al. (2009). A possible explanation for the higher pair fractions (of a few percent) found in higher mass galaxies is the stronger clustering of massive galaxies, which was not considered when the contamination due to projection effects was estimated. Another factor to consider is the small sample size of $\lesssim 89$ galaxy pairs, compared to our sample of $>1100$ mergers. That being said the trend found in this work is still one of only moderate significance, stressing the need for even larger samples to better characterize the dependence of GIF on host galaxy stellar mass.

\section{GALAXY MAJOR MERGER RATE}

The role mergers play in the formation and evolution of galaxies is largely unknown. The rate in which galaxies merge can affect the mass function of galaxies, and is likely linked at some level to the decline of the cosmic star formation rate. In this section, we discuss the galaxy major merger rate derived from the CFHTLS-Deep Catalog of Interacting Galaxies using Equation (2)

$$
\Re_{\mathrm{mg}}=\mathrm{GIF} / T_{\mathrm{mg}},
$$

where GIF is the galaxy interaction fraction, and $T_{\mathrm{mg}}$ is the assumed merger timescale. This equation provides a measure of the number of mergers galaxy ${ }^{-1} \mathrm{Gyr}^{-1}$. A primary source of uncertainty for all merger rate studies is the merger timescale. This observability timescale is essentially the length of the merger process over which a specific technique (e.g., finding close galaxy pairs or using morphology) is able to identify the galaxy as a merger. Estimates of merger timescales have been derived using dynamical friction arguments (Patton et al. 2002) as well galaxy scale numerical simulations tracing stellar or gas particles (Conselice 2006; Bell et al. 2006a; Iono et al. 2004).

Recent work by Lotz et al. (2008b), in which a morphological analysis was performed on a suite of $N$-body/hydrodynamical equal mass gas-rich mergers that have been processed through a radiative transfer code have provided reasonable estimates (0.2-0.9 Gyr), for the timescales probed by close pair methods and quantitative morphological parameters, such as $G-M_{20}$ and asymmetry. Lotz et al. (2008b) shows that quantitative morphological classifications based on $G-M_{20}$ are sensitive during the first encounter and final merger stages for gasrich equal mass mergers, but do not identify many interacting galaxies between these two stages, resulting in an observability (or merger) timescale range of 0.2-0.6 Gyr. Tidal tails can remain visible even after the central portion of the galaxy exhibits a uniform or symmetric appearance, therefore the timescale our technique is able to detect mergers is longer than that of $G-M_{20}$.

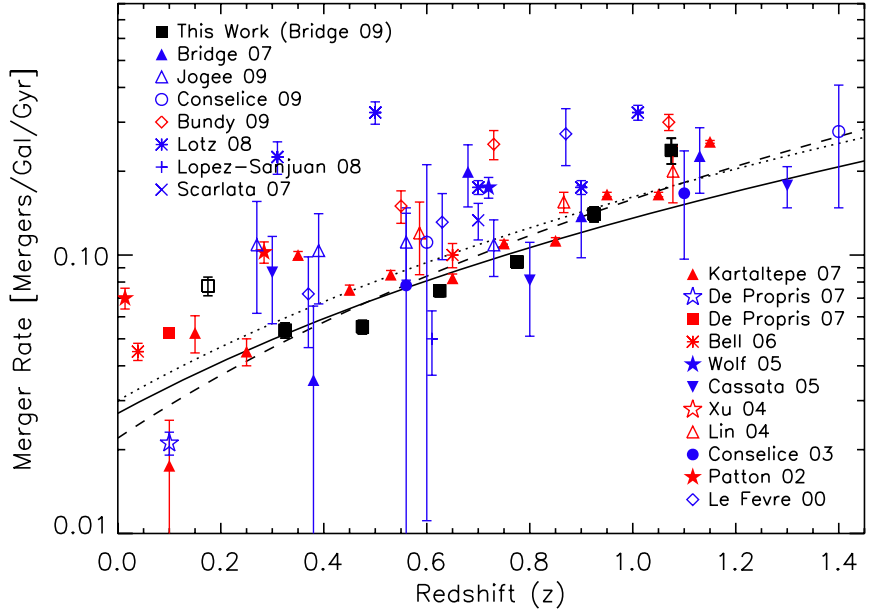

Figure 11. Merger rate as a function of redshift in units of mergers galaxy ${ }^{-1}$ $\mathrm{Gyr}^{-1}$. The filled black squares represent the merger rate derived using interacting galaxies with stellar masses $\geqslant 10^{9.5}\left(M_{\odot}\right)$. The best fit to our CFHTLS data (solid line) of the form $(1+z)^{m}$ finds $m=2.33 \pm 0.72, \operatorname{GIF}(0)=$ $0.027 \pm 0.003$. Combining the CFHTLS-Deep data with the other works noted below results in a best fit with $m=2.83 \pm 0.29$ (dashed line) and $m=2.43$ when a $2.0 \%$ statistical error is assumed. All fits include both the uncertainties of the merger fractions and merger timescale. The red points show the observed merger rate derived from close pair fractions as a function of redshift from Patton et al. (2002, filled stars), Lin et al. (2004, open upward triangles), Xu et al. (2004, open star), Bell et al. (2006b, lined star), De Propris et al. (2007, filled square), Kartaltepe et al. (2007, filled upward triangles), and Bundy et al. (2009, open diamonds). The merger rate derived from morphological studies as a function of redshift is shown in blue from Le Fèvre et al. (2000, open diamonds), Conselice et al. (2003, filled circle), Cassata et al. (2005, filled downward triangles), Wolf et al. (2005, open upward star), De Propris et al. (2007, open star), Scarlata et al. (2007, “x”), Bridge et al. (2007, open square), Lotz et al. (2008b, lined stars), Conselice et al. (2008, open circle), López-Sanjuan et al. (2009, plus sign), Jogee et al. (2009, open upward triangle), and this work (filled black squares). The assumed merger timescale for merger fractions derived using CAS or concentration, asymmetry was $0.9 \pm 0.2$ Gyr from Lotz et al. (2008a) and Conselice et al. (2008). The timescale over which mergers selected via $G-M_{20}$ is assumed to be $0.4 \pm 0.2 \mathrm{Gyr}$, close galaxy pairs $0.2 \pm 0.1 \mathrm{Gyr}$ (Lotz et al. 2008a), and this work $0.8 \pm 0.2 \mathrm{Gyr}$.

In order to estimate the merger timescale probed by visually identified mergers based on tidal features, we utilized $\mathrm{N}$-body simulations of galaxies undergoing mergers described in Conselice (2006) which employs the models of Mihos \& Hernquist (1996) and Mihos (2001). We carefully considered the duration that a galaxy encounter would exhibit the tidal features used in this work to identify interacting galaxies. We visually inspected snapshots of a simulated merger noting the duration in which the galaxies would be classified as "interacting" according to our criteria. Ultimately, we estimate the timescale being probed by strong visual tidal features to be $0.8 \pm 0.2$ Gyr.

Using Equation (2), the merger rate for galaxies with stellar masses $\geqslant 10^{9.5} M_{\odot}$ was derived and shown in Figure 11 as a function of redshift. The large uncertainty in the merger timescale reflects the various possible merger scenarios (i.e., large mass ratios can extend the merger timescale).

We find an average merger rate of $R_{\mathrm{mg}} \sim 0.065$ mergers $\mathrm{gal}^{-1} \mathrm{Gyr}^{-1}$ between $0.1 \leqslant z \leqslant 0.7$, which increases to 0.24 mergers gal $^{-1} \mathrm{Gyr}^{-1}$ at $z \sim 1.0$. The merger rate evolves with redshift as $(1+z)^{2.25 \pm 0.23}$. When both the Poisson errors in the GIF and the uncertainty in the merger timescale are included $m=2.33 \pm 0.72$.

Using our GIF results and the merger timescale derived above, we calculate a lower limit for the interaction history for typical 


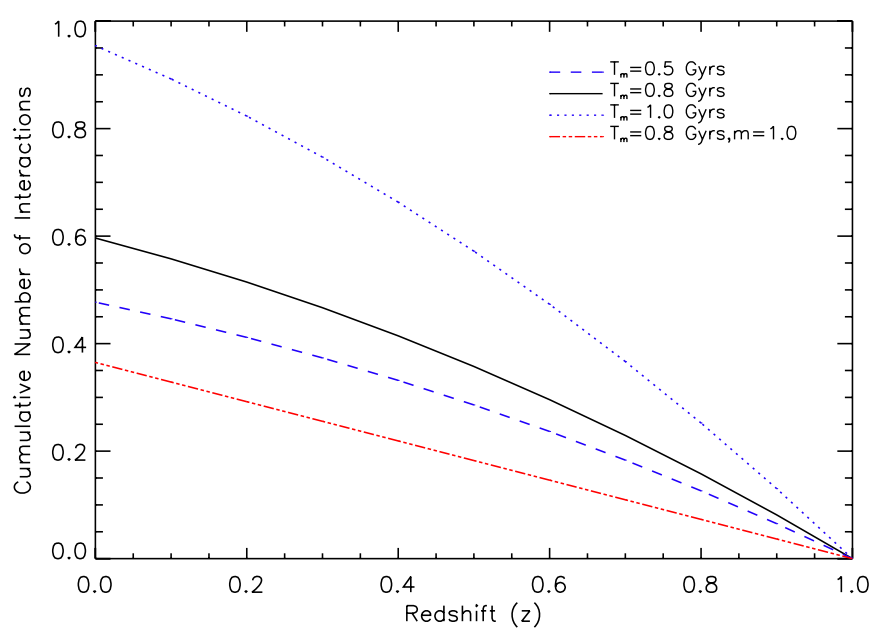

Figure 12. Interaction history, or the number of interactions an average galaxy in our sample has undergone since $z \sim 1$ (solid, black line). The coloured lines represent the same measurement but assume a different merger timescale, as stated in the plots legend. The assumed evolution of the merger rate was $(1+z)^{2.24 \pm 0.24}$. The red (dash-dotted) line is presented for comparison purposes assuming little evolution of the merger rate.

(A color version of this figure is available in the online journal.)

galaxies in our sample. Equation (3) (Conselice 2006) shows that by integrating the fraction of galaxies undergoing an interaction divided by the merger timescale one can obtain the number of interactions an average galaxy undergoes between two points in redshift space.

$$
n_{\mathrm{int}}=\int_{z_{2}}^{z_{1}} \frac{\mathrm{GIF}(z)}{T_{\mathrm{mg}}} d t=\int_{z_{2}}^{z_{1}} t_{H}\left(\frac{\mathrm{GIF}_{0}}{T_{\mathrm{mg}}}\right)(1+z)^{m-1} \frac{d z}{E(z)},
$$

where $t_{H}$ is a Hubble time, $\operatorname{GIF}(z)$ is the galaxy interaction fraction at a given redshift, $\mathrm{GIF}_{0}$ is the GIF at $z \sim 0$, and $E(z)=\left(\Omega_{M}(1+z)^{3} \Omega_{\Lambda}\right)^{-1 / 2}$. A power-law increase for the interaction rate was assumed, as it is well fit by the data.

Based on the above equation, and assuming $m=$ $2.25, \mathrm{GIF}_{0}=0.0215$ (the best fit to our data outlined in Section 4), we find that a galaxy with a stellar mass $\geqslant 10^{9.5} M_{\odot}$ (average mass ratio $>4: 1$ ) experiences $\sim 0.6$ mergers from $z=1.0$ to the present day (see Figure 12). Also shown in Figure 12 is the number of major mergers since $z \sim 1$ derived using a range of merger timescales.

\subsection{Merger Rate: Comparison with Previous Works}

As discussed in Section 4.3, a direct comparison between close pair fractions and morphological based merger fractions is only robust when the measurements are converted into a merger rate through the normalization of the appropriate observability timescale. Figure 11 presents the merger rate derived from CFHTLS-Deep, and compares it with previous studies. The assumed merger timescales were taken from Lotz et al. (2008a), where $G-M_{20}$ has an observability timescale of $0.4 \pm 0.2 \mathrm{Gyr}$, asymmetry (CAS) $0.9 \pm 0.2 \mathrm{Gyr}$, and the close pair selection, $0.2 \pm 0.1$ Gyr.

We find that the merger rate evolves as $m=2.33 \pm$ $0.72, G I F(0)=0.027 \pm 0.003$. Combining the CFHTLS-Deep data with the literature (Figure 11) results in a best fit with $m=2.83 \pm 0.29, \mathrm{GIF}(0)=0.022 \pm 0.003$ (dashed line). Since the fit is driven by data with small statistical errors, we also performed a fit assuming a standard $2 \%$ error, resulting in $m=2.43$. All fits include both the uncertainties of the merger fractions and the merger timescale. There is striking overall agreement in the literature supporting an increasing merger rate from the local universe to $z=1.3$. In the previous literature, it is often suggested that the various selection techniques (e.g., close pair versus morphology) are a source of discrepancy in terms of measuring the evolution merger rate evolution. Figure 11 shows that studies in which the close pair method was used to identify galaxy mergers (red data points) generally agree with morphologically based methods (blue points).

When considering the value of $m$ derived by close pair studies, our findings are in good agreement with Burkey et al. (1994; $m=2.5 \pm 0.5)$, Patton et al. (2002; $m=2.3 \pm 0.7)$, and Le Fèvre et al. (2000; $m=2.7 \pm 0.6)$. The largest close pair sample to date Kartaltepe et al. (2007) uses the 2 square degree COSMOS field, within which our D2 field is embedded. The value of $m$ derived using close pairs in COSMOS is mildly steeper than that found in our full sample at $m=$ $2.8 \pm 0.1$, but is consistent with our findings especially when we exclusively use the D2/COSMOS field (see Figure 5, showing cosmic variance). It should also be noted that Kartaltepe et al. (2007) use photometric redshifts to select galaxies separated by $<20 \mathrm{kpc}$, resulting in a larger contamination of non-merging pairs comparing to spectroscopic samples. Since the accuracy of their photometric redshifts evolves as $0.03(1+z)$, close pair fractions at higher redshift suffer larger contamination which would drive the evolution of the close pair fraction higher, which may explain their steeper value of $m$.

Our results do differ however from the close pair study of Lin et al. (2004; $m=1.08 \pm 0.4$ ) and morphological investigation of Lotz et al. (2008b; $m<1$; as discussed in Section 4.3) both of which utilized the DEEP2 survey. The merger rate derived using the close pair fractions of Lin et al. (2004) is consistent with the merger rate values found in this work and many others; however, they interpret their results as evidence for a flat merger rate evolution. This disparity mainly stems from the inclusion of lower redshift pair fractions from Patton et al. (2000, 2002), which are higher than other pair fractions in this redshift range, when fitting the equation, $(1+z)^{m}$. Another possible source of the discrepancy is that Lin et al. (2004) find that the value of $m$ changes by a factor of $\sim 4$ with the assumed luminosity evolution $(Q=0-1)$, we find no strong dependence of $m$ on $Q$ (Section 4.6). Finally, the small sample sizes are largely unable to statistically decipher between mild and moderate merger evolution $(1<m<2)$; however, the CFHTLS catalog having a sample $\sim 15$ times larger is not plagued by this specific uncertainty.

\section{STAR FORMATION RATES OF INTERACTING GALAXIES}

Interactions and collisions can profoundly affect the evolution of galaxies, through morphological transformations, mass accretion, and perhaps the most brilliant, through induced star formation. Observations of interacting galaxies such as the Antennae (Schweizer 1982) and simulations of major mergers (Mihos \& Hernquist 1996; Cox et al. 2006) both provide evidence that interactions can trigger violent starbursts.

As a first step in studying the impact mergers may have on a galaxies luminosity, we explored the $M_{G}$ distribution for four primary interacting galaxy types and compared them to the average $M_{G}$ magnitude for the non-interacting field population $\left(M_{G}=-20.66\right.$, Figure 13$)$. The four interacting types all show a higher average $M_{G}$ of $0.15-0.5 \mathrm{mag}$, compared to the 


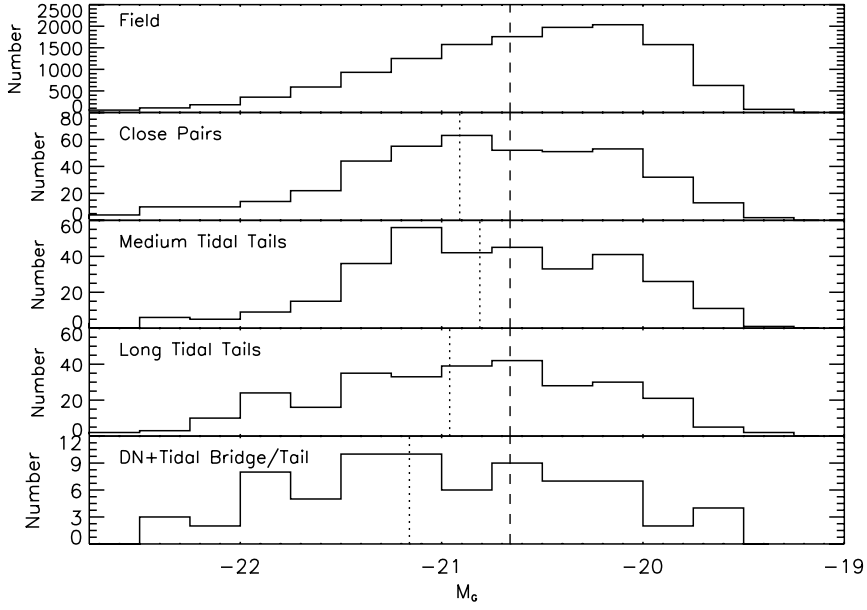

Figure 13. Absolute $g^{\prime}$-band magnitude $\left(M_{G}\right)$ distribution for galaxies classified as interacting and the field. The dotted line represents the average $M_{G}$ for each interacting class. Top: field galaxies (interacting galaxies removed) (second, third, fourth from top) show galaxies in a paired system with a tidal bridge, and galaxies with medium and long length tidal tails (bottom) are galaxies with a double nuclei and tidal features. The dashed line defines the average $M_{G}$ for the non-interacting field population. Luminosity evolution of $Q=1$ was assumed.

non-interacting population $\left(M_{G}=-20.81\right.$ for medium tidal tails, -20.91 for close pairs, -20.96 for long tails, and -21.16 for double nuclei with tidal tails), suggesting merger triggered star formation.

Taking a step further we used the five-band optical photometry of CFHTLS-Deep and derived SFRs for each galaxy in our sample (see Section 2.2). In Figure 14, we show the average SFR for each interaction class and the non-interacting field population as a function of redshift. All interaction classes from close pairs with tidal bridges (early stage mergers) to galaxies with double nuclei and tidal tails (later stage) exhibit enhanced SFRs a factor of 1.5-4 times that of non-interacting galaxies. The level of star formation enhancement also grows with the redshift. At higher redshifts, one might expect the average SFR to be larger simply because more gas is available. We do find that the average SFR for field galaxies (which is largely comprised of spiral or disk dominated galaxies $(>60 \%)$ ) increases marginally with redshift from $\sim 1.4 M_{\odot} \mathrm{yr}^{-1}$ at $z \sim 0.2$ to $\sim 3.2 M_{\odot} \mathrm{yr}^{-1}$ at $z \sim 0.75$. For interacting galaxies, however, we see a factor of 3 growth in the average SFR with redshift. This increase could be a result of these systems being more gas-rich, allowing tidally triggered starbursts to be more significant at higher redshifts.

Figure 14 also shows that galaxies in a close pair linked by a tidal tail (an early interaction stage), have SFRs similar to field galaxies, while in later stage mergers/galaxies with a tidal tail or double nuclei show the most enhancement, especially at $z>0.6$. These observations agree with $N$-body simulations which suggest that an initial starburst occurs after the first encounter, but typically a larger burst follows at the end of the merger sequence (Mihos \& Hernquist 1996; Hopkins et al. 2005).

\section{SUMMARY}

Using the deep five-band optical photometry from 2 square degrees of CFHTLS-Deep, we developed a new classification scheme to identify galaxy mergers based on the presence of strong tidal features. We visually classified $\approx 27,000$ galaxies, compiling the largest catalog of interacting galaxies $(\approx 1600)$ in

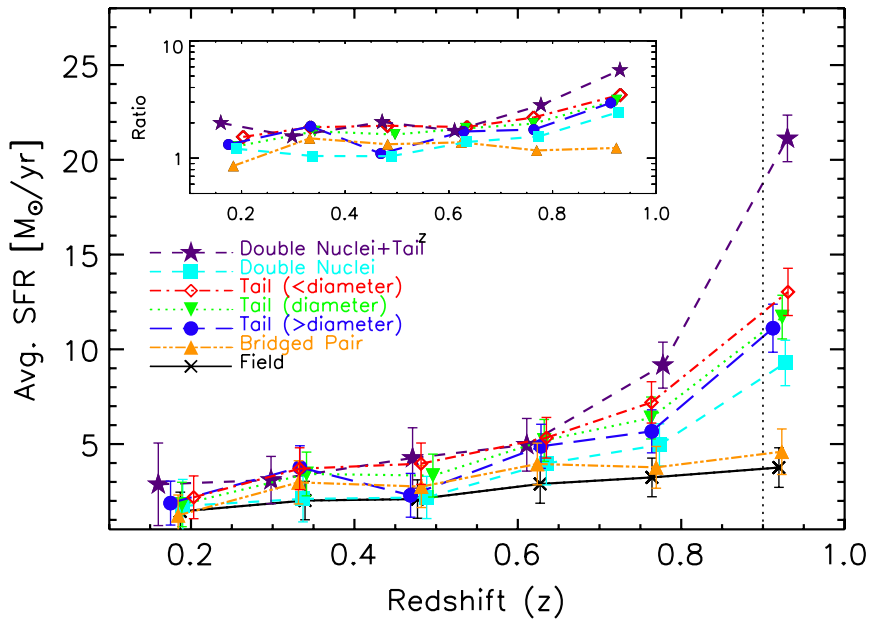

Figure 14. Average star formation rates for various interaction classes, as well as the field population. Inset is the ratio of the average SFR of a particular class compared to the field. The field is shown by the " $x$ ""s (black), bridged close pairs by triangle (orange), long tidal tails, circle (blue), intermediate tails, downward triangle (green), short tails, open diamond (red), double nuclei, square (cyan), and double nuclei with tail(s) are shown by stars (purple). The errors bars are the standard deviation of the SFRs.

(A color version of this figure is available in the online journal.)

the current literature. With this catalog we examine the redshift evolution of the galaxy merger fraction for galaxies with stellar masses of $M>10^{9.5} M_{\odot}$, and the impact merging has on star formation. Our results can be summarized as follows.

1. We find the galaxy merger fraction to be $4.3 \% \pm 0.3 \%$ at $z \sim 3$ and $19.0 \% \pm 2.5 \%$ at $z \sim 1$, implying that the frequency of galaxy interactions evolves with redshift as $(1+z)^{2.25 \pm 0.24}$. This evolution is a lower limit. A mild to nonevolving merger fraction $(m<1.5)$ with redshift is ruled out at the $\gtrsim 4 \sigma$ confidence level. This merger fraction study has the largest sample to date, a factor of $\gtrsim 10$ compared to previous work.

2. The implied average merger rate between $0.2<z<$ 0.6 is 0.075 mergers $\mathrm{gal}^{-1} \mathrm{Gyr}^{-1}$, which increases to 0.24 mergers gal $^{-1} \mathrm{Gyr}^{-1}$ at $z \sim 1$. Assuming $m=2.25$, a galaxy with a stellar mass $\geqslant 10^{9.5} M_{\odot}$ (and average mass ratio $4: 1$ ) experiences $\sim 0.6$ mergers from $z=1.0$ to the present day.

3. There is a moderately significant dependence of the merger fraction on galaxy stellar mass. We find that lower mass galaxies with $M_{*}<10^{10} M_{\odot}$ are more likely to be undergoing a merger than more massive systems $\left(M_{*}>\right.$ $\left.10^{10.7} M_{\odot}\right)$. This result is consistent with expectations of galaxy assembly downsizing.

4. A compilation of this work with previous merger rate studies presents an evolving merger rate, $\Re_{\mathrm{mg}}=\Re_{0}(1+z)^{m}$, with $m=2.83 \pm 0.29, \Re_{0}=0.022 \pm 0.003$ (errors include merger timescale uncertainty). Also noted is the general overall agreement between close pair and morphological selection techniques with respect to the galaxy merger rate and its evolution with redshift.

5. We find that major galaxy mergers have enhanced SFRs a factor of 2 higher than non-interacting galaxies. Late stage mergers show the largest enhancement, consistent with $N$ body simulations (Mihos \& Hernquist 1996; Hopkins et al. 2005 , to name a few). 
Overall we have presented a consistent picture of galaxy evolution whereby galaxy interactions occurred more frequently in the past, typically triggering bursts of star formation. These results show that tidal tails and bridges can be a powerful and robust tool in quantifying the interaction and merger rate evolution of galaxies. However, it must be stressed that the merger frequencies presented in this work are lower limits, and the fraction of galaxies undergoing a major merger or interaction is indeed higher, especially at larger redshifts.

Another consideration is that much of the star formation in the universe is enshrouded by dust. Optical estimates of SFRs can underestimate the effect merging has on triggering star formation in dusty systems (C. R. Bridge et al. 2010, in preparation). It has been shown that dusty, luminous IR galaxies (LIRGs) at $z>0.7$ are the dominant population contributing to the comoving infrared energy density and represent $\sim 70 \%$ of the star formation activity at $z \sim 1$ (Le Floc'h et al. 2005). So in order to compile a more complete picture of the role merging plays in driving the CSFR it is imperative that the processes involved in producing stars in these IR bright galaxies be understood. We are exploring the role merging plays in LIRGs at $0.5<1.0$ in a companion paper (C. R. Bridge et al. 2010 , in preparation). Clearly, more work with larger samples is needed to establish the merger rate at redshifts $>1$. Large survey areas with near-IR observations like those that will be provided by $H S T^{\prime}$ s Wide Field Camera 3 will provide key data sets. At low redshift $(<0.1<z<0.3)$, the merger rate is also poorly constrained and will benefit from future large area, deep, multi-band surveys such as the Large Synoptic Survey Telescope (LSST).

We thank Kevin Bundy, C. Borys, and Lee Armus for their contributions to this work and the anonymous referee for valuable comments that improved the clarity of the paper. Financial support for this work was provided in part by the Natural Sciences and Engineering Research Council (NSERC) and an Ontario Graduate Scholarship in Science and Technology. M.S. acknowledges support from the Royal Society. This paper is based on observations obtained with MegaPrime/ MegaCam, a joint project of CFHT and CEA/DAPNIA, at the Canada-France-Hawaii Telescope (CFHT). The authors recognize and acknowledge the very significant cultural role and reverence that the summit of Mauna Kea has always had within the indigenous Hawaiian community. We are most fortunate to have the opportunity to conduct observations from this mountain.

\section{REFERENCES}

Abraham, R. G., Tanvir, N. R., Santiago, B. X., Ellis, R. S., Glazebrook, K., \& van den Bergh, S. 1996a, MNRAS, 279, L47

Abraham, R. G., van den Bergh, S., Glazebrook, K., Ellis, R. S., Santiago,

B. X., Surma, P., \& Griffiths, R. E. 1996b, ApJS, 107, 1

Arp, H. 1966, ApJS, 14, 1

Barnes, J. E. 1992, ApJ, 393, 484

Barton, E. J., Geller, M. J., \& Kenyon, S. J. 2000, ApJ, 530, 660

Baum, W. A. 1962, in IAU Symp. 15, Problems of Extra-Galactic Research, ed. G. C. McVittie (New York: Macmillan), 390

Bell, E. F., Phleps, S., Somerville, R. S., Wolf, C., Borch, A., \& Meisenheimer, K. 2006b, ApJ, 652, 270

Bell, E. F., et al. 2006a, ApJ, 640, 241

Berrier, J. C., Bullock, J. S., Barton, E. J., Guenther, H. D., Zentner, A. R., \& Wechsler, R. H. 2006, ApJ, 652, 56

Bertin, E., \& Arnouts, S. 1996, A\&AS, 117, 393

Bolzonella, M., Miralles, J.-M., \& Pelló, R. 2000, A\&A, 363, 476
Boulade, O., et al. 2003, in Proc. SPIE, 4841, 72

Bridge, C. R., et al. 2007, ApJ, 659, 931

Bronder, T. J., et al. 2008, A\&A, 477, 717

Bundy, K., Fukugita, M., Ellis, R. S., Kodama, T., \& Conselice, C. J. 2004, ApJ, 601, L123

Bundy, K., Fukugita, M., Ellis, R. S., Targett, T. A., Belli, S., \& Kodama, T. 2009, ApJ, 697, 1369

Burkey, J. M., Keel, W. C., Windhorst, R. A., \& Franklin, B. E. 1994, ApJ, 429, L13

Carlberg, R. G., Pritchet, C. J., \& Infante, L. 1994, ApJ, 435, 540

Carlberg, R. G., Yee, H. K. C., Morris, S. L., Lin, H., Hall, P. B., Patton, D. R., Sawicki, M., \& Shepherd, C. W. 2001, ApJ, 563, 736

Carlberg, R. G., et al. 2000, ApJ, 532, L1

Cassata, P., et al. 2005, MNRAS, 357, 903

Conselice, C. J. 2006, ApJ, 638, 686

Conselice, C. J., Bershady, M. A., Dickinson, M., \& Papovich, C. 2003, AJ, 126,1183

Conselice, C. J., Bershady, M. A., \& Jangren, A. 2000, ApJ, 529, 886

Conselice, C. J., Rajgor, S., \& Myers, R. 2008, MNRAS, 386, 909

Conselice, C. J., Yang, C., \& Bluck, A. F. L. 2009, MNRAS, 394, 1956

Cox, T. J., Jonsson, P., Primack, J. R., \& Somerville, R. S. 2006, MNRAS, 373, 1013

De Propris, R., Conselice, C. J., Liske, J., Driver, S. P., Patton, D. R., Graham, A. W., \& Allen, P. D. 2007, ApJ, 666, 212

Dubinski, J., Mihos, J. C., \& Hernquist, L. 1999, ApJ, 526, 607

Fakhouri, O., \& Ma, C.-P. 2008, MNRAS, 386, 577

Fioc, M., \& Rocca-Volmerange, B. 1997, A\&A, 326, 950

Gottlöber, S., Klypin, A., \& Kravtsov, A. V. 2001, ApJ, 546, 223

Grazian, A., et al. 2006, A\&A, 449, 951

Gwyn, S. D. J., \& Hartwick, F. D. A. 1996, ApJ, 468, L77

Hopkins, P. F., Hernquist, L., Cox, T. J., Di Matteo, T., Martini, P., Robertson, B., \& Springel, V. 2005, ApJ, 630, 705

Hopkins, P. F., Hernquist, L., Cox, T. J., \& Kereš, D. 2008, ApJS, 175, 356

Howell, D. A., et al. 2005, ApJ, 634, 1190

Hubble, E. P. 1926, ApJ, 64, 321

Iono, D., Yun, M. S., \& Mihos, J. C. 2004, ApJ, 616, 199

Jogee, S., et al. 2009, ApJ, 697, 1971

Joye, W. A., \& Mandel, E. 2003, in ASP Conf. Ser. 295, Astronomical Data Analysis Software and Systems XII, ed. H. E. Payne, R. I. Jedrzejewski, \& R. N. Hook (San Francisco, CA: ASP), 489

Kampczyk, P., et al. 2007, ApJS, 172, 329

Kartaltepe, J. S., et al. 2007, ApJS, 172, 320

Le Borgne, D., \& Rocca-Volmerange, B. 2002, A\&A, 386, 446

Le Borgne, D., Rocca-Volmerange, B., Prugniel, P., Lançon, A., Fioc, M., \& Soubiran, C. 2004, A\&A, 425, 881

Le Fèvre, O., et al. 2000, MNRAS, 311, 565

Le Floc'h, E., et al. 2005, ApJ, 632, 169

Leauthaud, A., et al. 2007, ApJS, 172, 219

Lilly, S. J., Le Fèvre, O., Hammer, F., \& Crampton, D. 1996, ApJ, 460, L1

Lin, H., Yee, H. K. C., Carlberg, R. G., Morris, S. L., Sawicki, M., Patton, D. R., Wirth, G., \& Shepherd, C. W. 1999, ApJ, 518, 533

Lin, L., et al. 2004, ApJ, 617, L9

Loh, E. D., \& Spillar, E. J. 1986, ApJ, 303, 154

López-Sanjuan, C., et al. 2009, ApJ, 694, 643

Lotz, J. M., Jonsson, P., Cox, T. J., \& Primack, J. R. 2008a, MNRAS, 391, 1137

Lotz, J. M., Primack, J., \& Madau, P. 2004, AJ, 128, 163

Lotz, J. M., et al. 2008b, ApJ, 672, 177

Madau, P., Pozzetti, L., \& Dickinson, M. 1998, ApJ, 498, 106

Maller, A. H., Katz, N., Kereš, D., Davé, R., \& Weinberg, D. H. 2006, ApJ, 647, 763

McCarthy, P. J., et al. 2004, ApJ, 614, L9

Metcalfe, N., Shanks, T., Campos, A., McCracken, H. J., \& Fong, R. 2001, MNRAS, 323, 795

Mihos, J. C. 2001, ApJ, 550, 94

Mihos, J. C., \& Hernquist, L. 1996, ApJ, 464, 641

Patton, D. R., \& Atfield, J. E. 2008, ApJ, 685, 235

Patton, D. R., Carlberg, R. G., Marzke, R. O., Pritchet, C. J., da Costa, L. N., \& Pellegrini, P. S. 2000, ApJ, 536, 153

Patton, D. R., Pritchet, C. J., Yee, H. K. C., Ellingson, E., \& Carlberg, R. G. 1997, ApJ, 475, 29

Patton, D. R., et al. 2002, ApJ, 565, 208

Rana, N. C., \& Basu, S. 1992, A\&A, 265, 499

Sandage, A. 1961, The Hubble Atlas of Galaxies (Washington, DC: Carnegie Institution) 
Scarlata, C., et al. 2007, ApJS, 172, 406

Schweizer, F. 1982, ApJ, 252, 455

Sullivan, M., et al. 2006a, AJ, 131, 960

Sullivan, M., et al. 2006b, ApJ, 648, 868

Toomre, A. 1977, in Evolution of Galaxies and Stellar Populations, ed. B. M Tinsley \& R. B. Larson (New Haven, CT: Yale Univ. Observatory), 401

Toomre, A., \& Toomre, J. 1972, ApJ, 178, 623
Vorontsov-Velyaminov, B. A. 1959, Atlas and Catalog of Interacting Galaxies (Moscow: Sternberg Inst.)

Williams, R. E., et al. 1996, AJ, 112, 1335

Wolf, C., et al. 2005, ApJ, 630, 771

Xu, C. K., Sun, Y. C., \& He, X. T. 2004, ApJ, 603, L73

Yee, H. K. C., \& Ellingson, E. 1995, ApJ, 445, 37

Zepf, S. E., \& Koo, D. C. 1989, ApJ, 337, 34 